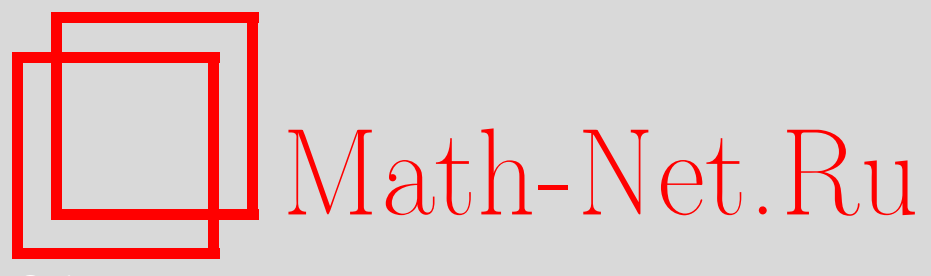

Х. А. Хачатрян, Об одном классе интегральных уравнений типа Урысона с сильной нелинейностью, Изв. РАН. Сер. матем., 2012, том 76, выпуск 1, 173-200

DOI: https://doi.org/10.4213/im5402

Использование Общероссийского математического портала Math-Net.Ru подразумевает, что вы прочитали и согласны с пользовательским соглашением http://www . mathnet.ru/rus/agreement

Параметры загрузки:

IP: 54.196 .121 .252

26 апреля 2023 г., 14:47:23

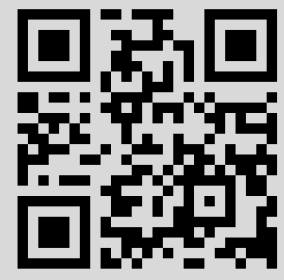




\title{
Х. А. Хачатрян \\ Об одном классе интегральных уравнений типа Урысона с сильной нелинейностью
}

\begin{abstract}
Исследуется класс однородных и неоднородных интегральных уравнений типа Урысона с сильной нелинейностью на положительной полупрямой. Предполагается, что нелинейный интегральный оператор типа Винера-Хопфа-Гаммерштейна служит локальной минорантой для соответствующего оператора Урысона. С помощью специальных методов линейной теории интегральных уравнений типа свертки построено положительное решение для указанных классов уравнений Урысона. Изучено также асимптотическое поведение построенного решения на бесконечности. В ходе доказательства этих утверждений в качестве вспомогательного факта строится однопараметрическое семейство положительных решений для нелинейных интегральных уравнений типа Винера-ХопфаГаммерштейна, соответствующий оператор которых служит минорантой для исходного оператора Урысона. Приведены частные примеры нелинейных интегральных уравнений, для которых выполняются все условия основных теорем.

Библиография: 19 наименований.
\end{abstract}

Ключевые слова: миноранта, уравнение Урысона, однопараметрическое семейство решений, факторизация.

\section{$\S 1$. Введение}

Как известно, вопросам построения положительных решений для нелинейных интегральных уравнений типа Урысона и Гаммерштейна посвящены исследования многих авторов, в частности М. А. Красносельского, П. П. Забрейко, В.Я. Стеценко (см. работы [1]-[7] и ссылки в них). В этом направлении разработан ряд эффективных методов решения задач, получены удобные в приложении признаки существования положительных решений для различных типов нелинейных интегральных уравнений Урысона или Гаммерштейна. Однако классическая теория, как правило, ограничивается исследованием тех нелинейных интегральных уравнений, соответствующий нелинейный оператор которых является компактным в рассматриваемых функциональных пространствах.

В последние годы в связи с развитием современной математической физики (в частности, в теории переноса излучения в спектральных линиях, $p$-адической математической физике, кинетической теории газов) возрос интерес к исследованию нелинейных интегральных уравнений Урысона с некомпактными интегральными операторами. В работах автора [8]-[11] рассмотрены разные нелинейные интегральные и интегро-дифференциальные уравнения с операторами Гаммерштейна и Урысона. Отличительной особенностью этих работ является отсутствие полной непрерывности соответствующего нелинейного интегрального оператора в определенных функциональных пространствах. 
В работе [8] исследуется нелинейное интегральное уравнение типа Урысона

$$
\varphi(x)=\int_{0}^{\infty} K(x, t, \varphi(t)) d t, \quad x \in(0,+\infty),
$$

в предположении, что существует число $\eta>0$ такое, что $\int_{0}^{\infty} K(x, t, \eta) d t \leqslant \eta$, $x \in(0,+\infty)$. В этой работе доказывается существование ограниченного решения уравнения (1.1) в случае, когда консервативное ядро Винера-Хопфа является локальной минорантой для оператора Урысона, т. е. $K(x, t, \tau) \geqslant K_{0}(x-t) \tau$.

Работа [9] посвящена изучению одного специального класса интегральных уравнений Гаммерштейна, имеющего конкретное применение в кинетической теории газов.

В работе [10] в предположении, что ядро Винера-Хопфа $K_{0}(x)$ представляет собой вполне монотонную четную функцию с особой структурой, а ядро Урысона удовлетворяет условию

$$
K(x, t, \tau) \geqslant K_{0}(x-t)(\tau-\omega(t+\tau)),
$$

где $\omega(x) \downarrow$ по $x$ на $[A,+\infty), A>0, \omega \in L_{1}\left(\mathbb{R}^{+}\right) \cap C\left(\mathbb{R}^{+}\right)$, а также условию

$$
\frac{1}{\delta(1+\theta x)} \int_{0}^{\infty} K(x, t, \delta(1+\theta t)) d t \leqslant 1, \quad x \in \mathbb{R}^{+}, \quad \theta, \delta>0, \quad \delta, \theta=\text { const }
$$

сформулированы теоремы существования положительного решения и исследовано асимптотическое поведение полученных решений на бесконечности.

Наконец, работа [11] посвящена изучению имеющего прикладное значение в теории нелокального взаимодействия волн класса нелинейных интегро-дифференциальных уравнений второго порядка с некомпактным оператором Урысона.

Настоящая работа посвящена исследованию однородных (1.1) и неоднородных

$$
H(x)=g(x)+\int_{0}^{\infty} K(x, t, H(t)) d t, \quad x \in(0,+\infty),
$$

нелинейных интегральных уравнений Урысона при более слабых ограничениях, налагаемых на ядро Урысона. Ядро $K(x, t, \tau)$, определенное на $(0,+\infty) \times$ $(0,+\infty) \times(-\infty,+\infty)$, является вещественной функцией, удовлетворяющей определенным условиям (см. теоремы 4.3, 4.4, а также теоремы 6.2, 6.3). Предполагается, что нелинейный интегральный оператор типа Винера-Хопфа-Гаммерштейна служит локальной минорантой для соответствующего оператора Урысона. В настоящей статье устанавливаются альтернативные теоремы о существовании положительных решений с помощью специальных методов линейной теории интегральных уравнений Винера-Хопфа и некоторых других методов теории функций, изучается асимптотическое поведение построенных решений на бесконечности. Оказывается, что однородное (1.1) и неоднородное (1.2) уравнения одновременно могут иметь положительные решения с одинаковым асимптотическим поведением. В качестве вспомогательного факта строится однопараметрическое семейство положительных решений для некоторых классов однородных и неоднородных интегральных уравнений без параметра типа Гаммерштейна. В конце работы приведены частные примеры интегральных уравнений Урысона, которые иллюстрируют доказанные теоремы. 


\section{§ 2. Некоторые вспомогательные факты из теории линейных интегральных уравнений типа свертки}

2.1. Об одном однородном линейном интегральном уравнении с суммарно-разностным ядром. Рассмотрим следующее однородное интегральное уравнение с суммарно-разностным ядром на полуоси:

$$
S(x)=\int_{0}^{\infty}\left[K_{0}(x-t)-\varepsilon K_{0}(x+t)\right] S(t) d t, \quad x \in(0,+\infty),
$$

относительно искомой функции $S(x)$. Здесь $K_{0}(x)$ - определенная на $(-\infty,+\infty)$ измеримая функция, причем

$$
\begin{gathered}
0<K_{0} \in L_{1}(\mathbb{R}), \quad \int_{-\infty}^{+\infty} K_{0}(\tau) d \tau=1, \quad K_{0}(-x)=K_{0}(x), \quad x \in(0,+\infty), \\
\nu_{j}\left(K_{0}\right) \equiv \int_{0}^{\infty} x^{j} K_{0}(x) d x<+\infty, \quad j=1,2,3, \\
K_{0}(x) \downarrow \quad \text { по } x \text { на }(0,+\infty),
\end{gathered}
$$

$\mathrm{a} \varepsilon \in[0,1]$ - заданный числовой параметр уравнения.

В настоящем пункте мы построим нетривиальное неотрицательное (а в случае $\varepsilon \in[0,1)$ - положительное) решение уравнения (2.1) и исследуем асимптотическое поведение решения на бесконечности. С этой целью введем несколько обозначений, сформулируем некоторые известные результаты из теории интегральных уравнений Винера-Хопфа в консервативном случае, а также докажем некоторые новые факты из этой теории.

Пусть $E$ - одно из следующих банаховых пространств: $L_{p}(0,+\infty), p \geqslant 1$, $M[0,+\infty), C_{M}[0,+\infty), C_{0}[0,+\infty)$, где $M[0,+\infty)$ - пространство ограниченных функций, определенных на $[0,+\infty), C_{M}[0,+\infty)$ - пространство непрерывных и ограниченных функции на интервале $[0,+\infty)$, а $C_{0}[0,+\infty)$ - пространство непрерывных функций на $[0,+\infty)$ с нулевым пределом на бесконечности. Обозначим через $\Omega$ класс интегральных операторов Винера-Хопфа: $\mathcal{K}_{0} \in \Omega$, если существует функция $K_{0} \in L_{1}(-\infty,+\infty)$ такая, что

$$
\left(\mathcal{K}_{0} f\right)(x)=\int_{0}^{\infty} K_{0}(x-t) f(t) d t, \quad f \in E .
$$

Как известно (см., например, [12]), операторы из $\Omega$ действуют в каждом из пространств $E$, причем

$$
\left\|\mathcal{K}_{0}\right\|_{E} \leqslant \int_{-\infty}^{+\infty}\left|K_{0}(\tau)\right| d \tau
$$

Однако операторы Винера-Хопфа не являются вполне непрерывными в банаховых пространствах $E$.

Пусть $\Omega_{ \pm} \subset \Omega-$ следующие алгебры пространства $\Omega: V_{ \pm} \in \Omega_{ \pm}$, если существуют функции $v_{ \pm} \in L_{1}(0,+\infty)$ такие, что

$$
\left(V_{-} f\right)(x)=\int_{x}^{\infty} v_{-}(t-x) f(t) d t, \quad\left(V_{+} f\right)(x)=\int_{0}^{x} v_{+}(x-t) f(t) d t, \quad f \in E .
$$


Нетрудно убедиться в том, что тогда $\Omega=\Omega_{+} \oplus \Omega_{-}$. Введем также следующий класс интегральных операторов типа Ганкеля: $\mathcal{K}_{1} \in \Omega_{1}$, если существует измеримая функция $K_{1} \in L_{1}(0,+\infty)$ такая, что

$$
\left(\mathcal{K}_{1} f\right)(x)=\int_{0}^{\infty} K_{1}(x+t) f(t) d t, \quad f \in E, \quad x \in(0,+\infty) .
$$

Заметим, что операторы из $\Omega_{1}$ непрерывно действуют в пространствах $E$ и, в отличие от операторов Винера-Хопфа, являются вполне непрерывными в $E$ (см. [13]).

Уравнение (2.1) запишем в операторной форме

$$
\left(I-\mathcal{K}_{0}+\varepsilon \mathcal{K}_{1}\right) S=0
$$

где $I$ - единичный оператор, $\mathcal{K}_{0} \in \Omega$ и $\left(\mathcal{K}_{0} f\right)(x)=\int_{0}^{\infty} K_{0}(x-t) f(t) d t, x \in$ $(0,+\infty), \mathcal{K}_{1} \in \Omega_{1}$ и $\left(\mathcal{K}_{1} f\right)(x)=\int_{0}^{\infty} K_{0}(x+t) f(t) d t, x \in(0,+\infty)$, причем ядро удовлетворяет условиям (2.2)-(2.4).

Как известно (см. [14]), оператор $I-\mathcal{K}_{0}+\varepsilon \mathcal{K}_{1}$ допускает следующую факторизацию:

$$
I-\mathcal{K}_{0}+\varepsilon \mathcal{K}_{1}=\left(I-V_{-}\right)(I+W)\left(I-V_{+}\right),
$$

где

$$
\begin{gathered}
V_{ \pm} \in \Omega_{ \pm}, \quad \text { причем } \quad 0 \leqslant v_{ \pm} \in L_{1}(0,+\infty), \quad \gamma_{ \pm}=\int_{0}^{\infty} v_{ \pm}(\tau) d \tau=1, \\
W \in \Omega_{1}, \quad(W f)(x)=\int_{0}^{\infty} W(x+t) f(t) d t, \quad W(x) \geqslant 0, \quad x \in \mathbb{R}^{+} \\
\tilde{m}_{1}(W) \equiv \int_{0}^{\infty} x W(x) d x<+\infty .
\end{gathered}
$$

С учетом факторизации (2.10) решение уравнения (2.1) сводится к последовательному решению следующих связанных уравнений:

$$
\begin{aligned}
& \left(I-V_{-}\right) S_{0}=0, \\
& (I+W) S_{1}=S_{0}, \\
& \left(I-V_{+}\right) S=S_{1} .
\end{aligned}
$$

Возможны следующие случаи:

a) $\lambda=-1$ не является собственным значением вполне непрерывного оператора $W$

b) $\lambda=-1$ является собственным значением $W$.

Случай а). Уравнение (2.14) запишем в раскрытом виде:

$$
S_{0}(x)=\int_{x}^{\infty} v_{-}(t-x) S_{0}(t) d t, \quad x>0 .
$$

Поскольку $\gamma_{-}=1$, то $S_{0}(x) \equiv 1$ будет удовлетворять уравнению (2.17). Подставляя это значение в $(2.15)$, приходим к неоднородному интегральному уравнению

$$
S_{1}(x)=1-\int_{0}^{\infty} W(x+t) S_{1}(t) d t, \quad x \in \mathbb{R}^{+},
$$


относительно искомой функции $S_{1}(x)$. В рассматриваемом случае уравнение (2.18) имеет ограниченное решение: $S_{1} \in M(0,+\infty)$. С учетом простой оценки

$$
\left|1-S_{1}(x)\right| \leqslant \sup _{x \in \mathbb{R}^{+}}\left|S_{1}(x)\right| \int_{x}^{\infty} W(\tau) d \tau \rightarrow 0, \quad x \rightarrow+\infty,
$$

из теоремы Фубини (см. [15]) следует, что

$$
\lim _{x \rightarrow+\infty} S_{1}(x)=1, \quad 1-S_{1} \in L_{1}(0,+\infty) \cap M(0,+\infty),
$$

так как выполняется условие (2.13). Перейдем теперь к решению уравнения (2.16) в следующей форме:

$$
S(x)=S_{1}(x)+\int_{0}^{x} v_{+}(x-t) S(t) d t, \quad x \in \mathbb{R}^{+} .
$$

Поскольку свободный член $S_{1}$ в $(2.21)$ удовлетворяет условиям $(2.20)$, то из результатов работы [16] следует, что уравнение (2.21) имеет решение в следующем виде:

$$
S(x)=\frac{1}{m_{1}} x+Q(x)
$$

где

$$
m_{1} \equiv \int_{0}^{\infty} x v_{+}(x) d x<+\infty, \quad Q \in M(0,+\infty) .
$$

Случай b). Из решений уравнения (2.17) выберем нулевое решение $S_{0} \equiv 0$. Подставляя это решение в (2.15), в рассматриваемом случае получаем уравнение

$$
S_{1}(x)=-\int_{0}^{\infty} W(x+t) S_{1}(t) d t, \quad x>0,
$$

которое имеет ненулевое ограниченное решение $S_{1}(x)$. Из оценки

$$
\left|S_{1}(x)\right| \leqslant \sup _{x \in \mathbb{R}^{+}}\left|S_{1}(x)\right| \int_{x}^{\infty} W(\tau) d \tau
$$

непосредственно следует, что $S_{1}(x) \rightarrow 0$, когда $x \rightarrow+\infty$, причем $S_{1} \in$ $L_{1}(0,+\infty)$, так как $\widetilde{m}_{1}(W)<+\infty$. Итак, $S_{1} \in L_{1}(0,+\infty) \cap M(0,+\infty)$. Из результатов, полученных в работе [16], сразу следует, что если $S_{1} \in L_{1}(0,+\infty) \cap$ $M(0,+\infty)$, то уравнение $(2.21)$ имеет ограниченное решение $S(x)$.

Отметим, что в случаях a) и b) полученное решение может быть знакопеременным.

В дальнейшем изложении настоящей работы нам существенно понадобится именно положительное решение уравнения (2.1). С этой целью докажем, что уравнение (2.1) обладает также положительным решением $S^{*}(x) \geqslant|S(x)|$, обладающим дополнительными свойствами.

ТеОрема 2.1. Предположим, что $\lambda=-1$ не является собственным значением оператора $W$ и выполнены условия (2.2)-(2.4). Тогда если $\varepsilon=1$, то 
уравнение (2.1) имеет ненулевое неотрицательное решение $S^{*}(x)$, причем

$$
\begin{gathered}
|S(x)| \leqslant S^{*}(x) \leqslant \frac{1}{m_{1}} x+\sup _{x \in \mathbb{R}^{+}}|Q(x)|, \\
S^{*}(x) \sim \frac{1}{m_{1}} x, \quad x \rightarrow+\infty .
\end{gathered}
$$

Более того, если $\varepsilon \in[0,1)$ u $l_{0} \equiv \sup _{x \in \mathbb{R}^{+}} \frac{\int_{x}^{\infty} K_{0}(\tau) d \tau}{K_{0}(x)}<+\infty$, то (2.1) имеет положительное монотонно возрастающее решение $S^{*}(x) \geqslant|S(x)|$, причем

$$
S^{*}(x) \sim \frac{1}{m_{1}} x, \quad x \rightarrow+\infty, \quad \inf _{x \in \mathbb{R}^{+}} S^{*}(x) \equiv \alpha_{0}>0, \quad S^{*}(x) \leqslant \frac{1}{m_{1}} x+c,
$$

əəe

$$
c \equiv \max \left(\frac{l_{0}}{m_{1}}, \frac{2 r_{0}}{m_{1}} \sup _{x \in \mathbb{R}^{+}}|Q(x)|\right), \quad r_{0}=\int_{0}^{\infty} t K_{0}(t) d t .
$$

ДокАзАТЕльство. Сначала подробно обсудим случай $\varepsilon=1$. Рассмотрим следующие последовательные приближения:

$$
\begin{gathered}
S^{(n+1)}(x)=\int_{0}^{\infty}\left[K_{0}(x-t)-K_{0}(x+t)\right] S^{(n)}(t) d t, \quad n=0,1,2, \ldots, \\
S^{(0)}(x)=\frac{1}{m_{1}} x+\sup _{x \in \mathbb{R}^{+}}|Q(x)|, \quad x>0
\end{gathered}
$$

По индукции докажем, что $S^{(n)}(x) \downarrow$ по $n$. Убедимся в том, что $S^{(1)}(x) \leqslant S^{(0)}(x)$ при всех $x>0$. С этой целью проверим неравенство

$$
\int_{0}^{\infty}\left[K_{0}(x-t)-K_{0}(x+t)\right]\left(\frac{1}{m_{1}} t+\sup _{x \in \mathbb{R}^{+}}|Q(x)|\right) d t \leqslant \frac{1}{m_{1}} x+\sup _{x \in \mathbb{R}^{+}}|Q(x)| .
$$

Из (2.2)-(2.4) следует, что

$$
\begin{aligned}
\int_{0}^{\infty} & {\left[K_{0}(x-t)-K_{0}(x+t)\right]\left(\frac{1}{m_{1}} t+\sup _{x \in \mathbb{R}^{+}}|Q(x)|\right) d t } \\
=\int_{-\infty}^{x} K_{0}(\tau)\left(\frac{1}{m_{1}}(x-\tau)+\sup _{x \in \mathbb{R}^{+}}|Q(x)|\right) d \tau & \quad-\int_{x}^{\infty} K_{0}(\tau)\left(\frac{1}{m_{1}}(\tau-x)+\sup _{x \in \mathbb{R}^{+}}|Q(x)|\right) d \tau \\
= & \frac{1}{m_{1}} x+\sup _{x \in \mathbb{R}^{+}}|Q(x)|-2 \sup _{x \in \mathbb{R}^{+}}|Q(x)| \int_{x}^{\infty} K_{0}(\tau) d \tau \leqslant \frac{1}{m_{1}} x+\sup _{x \in \mathbb{R}^{+}}|Q(x)| .
\end{aligned}
$$

Из последнего неравенства следует, что

$$
S^{(1)}(x) \leqslant S^{(0)}(x) .
$$

Поскольку $K_{0}(x-t) \geqslant K_{0}(x+t),(x, t) \in \mathbb{R}^{+} \times \mathbb{R}^{+}$, то, предполагая, что $S^{(n)}(x) \leqslant$ $S^{(n-1)}(x)$, из $(2.27)$ получим $S^{(n+1)}(x) \leqslant S^{(n)}(x)$. Теперь по индукции докажем, что

$$
S^{(n)}(x) \geqslant|S(x)|, \quad n=0,1,2, \ldots
$$


В случае $n=0$ это очевидно, поскольку

$$
|S(x)| \leqslant \frac{1}{m_{1}} x+\sup _{x \in \mathbb{R}^{+}}|Q(x)|=S^{(0)}(x) .
$$

Предположим теперь, что неравенство (2.28) выполняется при некотором $n \in \mathbb{N}$, и докажем его в случае $n+1$. Имеем

$$
S^{(n+1)}(x) \geqslant \int_{0}^{\infty}\left[K_{0}(x-t)-K_{0}(x+t)\right]|S(t)| d t \geqslant|S(x)| .
$$

Из неравенства $(2.28)$ в силу монотонности $S^{(n)}(x)$ по $n$ следует, что последовательность функций $\left\{S^{(n)}(x)\right\}_{n=0}^{\infty}$ имеет предел

$$
\lim _{n \rightarrow \infty} S^{(n)}(x)=S^{\sharp}(x) .
$$

Из предельной теоремы Б. Леви (см. [15]) следует, что $S^{\sharp}(x)$ удовлетворяет уравнению $(2.1)$, а из $(2.27),(2.28)$ получаем

$$
\frac{1}{m_{1}} x+\sup _{x \in \mathbb{R}^{+}}|Q(x)| \geqslant S^{\sharp}(x) \geqslant|S(x)|, \quad x>0 .
$$

Следовательно, учитывая (2.22), (2.30), можем утверждать, что

$$
S^{\sharp}(x) \sim \frac{1}{m_{1}} x, \quad x \rightarrow+\infty .
$$

Теперь докажем, что $S^{\sharp}(x) \uparrow$ по $x$ на $(0,+\infty)$. С этой целью сначала по индукции докажем, что

$$
S^{(n)}(x) \uparrow \quad \text { по } x \text { на }(0,+\infty), \quad n=0,1,2, \ldots .
$$

Действительно, записывая последовательные приближения (2.27) в виде

$$
\begin{gathered}
S^{(n+1)}(x)=\int_{-\infty}^{x} K_{0}(\tau) S^{(n)}(x-\tau) d \tau-\int_{x}^{\infty} K_{0}(\tau) S^{(n)}(\tau-x) d \tau \\
S^{(0)}(x)=\frac{1}{m_{1}} x+c, \quad n=0,1,2, \ldots
\end{gathered}
$$

и предполагая, что $S^{(n)}(x) \uparrow$ по $x$, из $(2.33)$ получаем, что $S^{(n+1)}(x) \uparrow$ по $x$. Таким образом, из последнего следует, что $S^{\sharp}(x) \uparrow$ по $x$.

Теперь рассмотрим случай, когда $\varepsilon \in[0,1)$. Докажем следующую вспомогательную лемму.

ЛЕмма 2.1. Пусть $l_{0} \equiv \sup _{x \in \mathbb{R}^{+}} \frac{\int_{x}^{\infty} K_{0}(\tau) d \tau}{K_{0}(x)}<+\infty$. Тогда при выполнении условии $(2.2)-(2.4)$ для всех $\varepsilon \in[0,1]$ справедливо неравенство

$$
\frac{1-\varepsilon}{m_{1} c(1+\varepsilon)+(1-\varepsilon) x} \leqslant \frac{\int_{x}^{\infty} K_{0}(t) d t}{\int_{x}^{\infty} t K_{0}(t) d t}, \quad x \in \mathbb{R}^{+},
$$

где число с задается согласно (2.26). 
ДокАЗАТЕЛьство. Введем следующую функцию:

$F(x)=\left(m_{1} c(1+\varepsilon)+(1-\varepsilon) x\right) \int_{x}^{\infty} K_{0}(t) d t-(1-\varepsilon) \int_{x}^{\infty} t K_{0}(t) d t, \quad x \in[0,+\infty)$.

Заметим, что

$$
F(0)=\frac{m_{1} c(1+\varepsilon)}{2}-(1-\varepsilon) r_{0}>0,
$$

поскольку $c>\frac{2 r_{0}}{m_{1}}$. С другой стороны, имеем

$$
F(+\infty)=0, \quad F^{\prime}(x)=(1-\varepsilon) \int_{x}^{\infty} K_{0}(t) d t-m_{1} c(1+\varepsilon) K_{0}(x) \leqslant 0, \quad x \in \mathbb{R}^{+},
$$

так как $c>\frac{l_{0}}{m_{1}}$. Из $(2.35),(2.36)$ следует $F(x) \geqslant 0$, что и доказывает лемму.

Рассмотрим теперь следующие последовательные приближения:

$$
\begin{gathered}
S^{(n+1)}(x)=\int_{0}^{\infty}\left[K_{0}(x-t)-\varepsilon K_{0}(x+t)\right] S^{(n)}(t) d t, \quad n=0,1,2, \ldots, \\
S^{(0)}=\frac{1}{m_{1}} x+c, \quad x \in \mathbb{R}^{+} .
\end{gathered}
$$

Используя (2.26) и (2.34), по индукции можно убедиться в достоверности следующих фактов:

$$
\begin{gathered}
S^{(n)}(x) \downarrow \quad \text { по } n ; \\
S^{(n)}(x) \uparrow \quad \text { по } x \text { на }(0,+\infty), \quad n=0,1,2, \ldots ; \\
S^{(n)}(x) \geqslant S^{\sharp}(x), \quad n=0,1,2, \ldots .
\end{gathered}
$$

Таким образом, последовательность функций $\left\{S^{(n)}(x)\right\}_{n=0}^{\infty}$ имеет предел

$$
\lim _{n \rightarrow \infty} S^{(n)}(x)=S^{*}(x) \geqslant S^{\sharp}(x),
$$

причем этот предел удовлетворяет уравнению (2.1), представляет собой монотонно возрастающую функцию и имеет асимптотику: $S^{*}(x) \sim \frac{1}{m_{1}} x$, когда $x \rightarrow+\infty$.

Докажем, что $\inf _{x \in \mathbb{R}^{+}} S^{*}(x) \equiv \alpha_{0}>0$. Поскольку $S^{*}(x) \geqslant S^{\sharp}(x), S^{\sharp}(x) \geqslant 0$, $S^{\sharp}(x) \not \equiv 0$, существует хотя бы одна точка $x_{0} \geqslant 0$ такая, что $S^{*}\left(x_{0}\right)>0$. Фиксируем эту точку. Из уравнения $(2.1)$ с учетом монотонности функции $S^{*}(x)$ получим

$$
\begin{aligned}
S^{*}(x) & \geqslant \int_{x_{0}}^{\infty}\left[K_{0}(x-t)-\varepsilon K_{0}(x+t)\right] S^{*}(t) d t \\
& \geqslant S^{*}\left(x_{0}\right)\left(\int_{-\infty}^{x-x_{0}} K_{0}(\tau) d \tau-\varepsilon \int_{x+x_{0}}^{\infty} K_{0}(\tau) d \tau\right) \\
& \geqslant S^{*}\left(x_{0}\right)\left(\int_{-\infty}^{-x_{0}} K_{0}(\tau) d \tau-\varepsilon \int_{x_{0}}^{\infty} K_{0}(\tau) d \tau\right) \\
& =(1-\varepsilon) S^{*}\left(x_{0}\right) \int_{x_{0}}^{\infty} K_{0}(\tau) d \tau>0
\end{aligned}
$$


откуда следует, что

$$
\alpha_{0} \geqslant(1-\varepsilon) S^{*}\left(x_{0}\right) \int_{x_{0}}^{\infty} K_{0}(\tau) d \tau>0 .
$$

Таким образом, теорема доказана.

Аналогично доказывается следующая

Теорема 2.2. Предположим, что $\lambda=-1$ является собственным значением оператора $W$ и выполнены условия (2.2)-(2.4). Тогда если $\varepsilon=1$, то уравнение (2.1) имеет ненулевое неотрицательное возрастающее ограниченное решение $S^{\sharp}(x)$, причем $|S(x)| \leqslant S^{\sharp}(x) \leqslant \sup _{x \in \mathbb{R}^{+}}|S(x)|$. Если $\varepsilon \in[0,1)$, то уравнение (2.1) имеет положительное монотонно возрастающее ограниченное решение $S^{*}(x) \geqslant S^{\sharp}(x)$, причем

$$
\begin{gathered}
S^{*}(x) \leqslant \sup _{x \in \mathbb{R}^{+}} S^{\sharp}(x), \quad x \in \mathbb{R}^{+}, \\
\inf _{x \in \mathbb{R}^{+}} S^{*}(x) \equiv \beta_{0}>0, \quad \lim _{x \rightarrow+\infty} S^{*}(x)=\sup _{x \in \mathbb{R}^{+}} S^{\sharp}(x) .
\end{gathered}
$$

2.2. Одно обобщение уравнения (2.1). В этом пункте рассмотрим вопросы построения положительного монотонно возрастающего решения для следующего более общего однородного интегрального уравнения:

$$
B(x)=\mu(x) \int_{0}^{\infty}\left[K_{0}(x-t)-\varepsilon K_{0}(x+t)\right] B(t) d t, \quad x \in \mathbb{R}^{+},
$$

относительно искомой функции $B(x)$, где $\mu(x)$ - определенная на $\mathbb{R}^{+}$измеримая функция, удовлетворяющая условиям

$$
\begin{gathered}
0<\mu_{0} \leqslant \mu(x) \leqslant 1, \quad x \in \mathbb{R}^{+}, \quad \mu(x) \uparrow \quad \text { по } x \text { на } \mathbb{R}^{+}, \\
(1-\mu(x)) x^{j} \in L_{1}\left(\mathbb{R}^{+}\right), \quad j=0,1 .
\end{gathered}
$$

Объединяя результаты, полученные в [17], с результатами из п. 2.1 настоящей работы, можем утверждать, что при условиях (2.2)-(2.4), (2.42) уравнение (2.41) имеет нетривиальное решение

$$
0 \leqslant B_{0}(x) \equiv S^{\sharp}(x)-\varphi(x) \leqslant S^{\sharp}(x) \quad\left(B_{0}(x) \not \equiv S^{\sharp}(x)\right),
$$

где $0 \leqslant \varphi(x) \leqslant S^{\sharp}(x)\left(\varphi(x) \not \equiv S^{\sharp}(x)\right)$ - ограниченное решение уравнения

$$
\varphi(x)=(1-\mu(x)) S^{\sharp}(x)+\mu(x) \int_{0}^{\infty} K_{0}(x-t) \varphi(t) d t, \quad x \in \mathbb{R}^{+} .
$$

С использованием неотрицательного решения $B_{0}(x)$ так же, как в п. 2.1, устанавливаются следующие результаты.

ТеОрема 2.3. Пусть $\lambda=-1$ не является собственным значением оператора $W$ и выполнены условия (2.2)-(2.4), (2.42). Тогда если $B(x) \geqslant B_{0}(x)$, то уравнение (2.41) имеет ненулевое неотрицательное монотонно возрастающее решение $B(x)$, причем

$$
\begin{gathered}
B(x) \leqslant \frac{1}{m_{1}} x+\sup _{x \in \mathbb{R}^{+}}|Q(x)|, \quad x \in \mathbb{R}^{+}, \\
B(x) \sim \frac{1}{m_{1}} x, \quad x \rightarrow+\infty .
\end{gathered}
$$


Далее, при $\varepsilon \in[0,1), l_{0}<+\infty$ уравнение (2.41) имеет положительное монотонно возрастающее решение $B^{*}(x) \geqslant B(x)$, причем

$$
\begin{gathered}
B^{*}(x) \sim \frac{1}{m_{1}} x, \quad x \rightarrow+\infty, \\
\inf _{x \in \mathbb{R}^{+}} B^{*}(x) \equiv \beta_{0}>0, \quad B^{*}(x) \leqslant \frac{1}{m_{1}} x+c,
\end{gathered}
$$

где число с задается согласно (2.26).

ТЕОрема 2.4. Предположим, что $\lambda=-1$ является собственным значением оператора $W$ и выполнены условия (2.2)-(2.4), (2.42). Тогда если $\varepsilon=1$, то уравнение (2.41) имеет ненулевое неотрицательное монотонно возрастающее ограниченное решение $B(x) \geqslant B_{0}(x)$. Если $\varepsilon \in[0,1)$, то уравнение (2.41) имеет положительное монотонно возрастающее ограниченное решение $B^{*}(x) \geqslant B(x)$, причем

$$
\begin{gathered}
B^{*}(x) \leqslant \sup _{x \in \mathbb{R}^{+}} B(x), \quad x \in \mathbb{R}^{+}, \\
\inf _{x \in \mathbb{R}^{+}} B^{*}(x) \equiv \beta_{0}>0, \quad \lim _{x \rightarrow+\infty} B^{*}(x)=\sup _{x \in \mathbb{R}^{+}} B(x) .
\end{gathered}
$$

\section{§ 3. Разрешимость уравнения (1.1) в случае линейной миноранты для оператора Урысона}

3.1. Формулировка результатов. Ниже, предполагая, что для исходного оператора Урысона минорантой служит линейный интегральный оператор с разностным ядром, рассмотрим случаи:

a) $\lambda=-1$ не является собственным значением оператора $W$;

b) $\lambda=-1$ является собственным значением оператора $W$.

В зависимости от случаев а) или b) относительно функции $K(x, t, \tau)$ сделаем следующие предположения.

Случай а). Пусть существует положительное число $\delta>0$ такое, что:

1) $\frac{1}{\frac{1}{m_{1}} x+c} \int_{0}^{\infty} K\left(x, t, \delta\left(\frac{1}{m_{1}} t+c\right)\right) d t \leqslant \delta, x \in \mathbb{R}^{+}$

2) $K \in \operatorname{Carat}\left(\mathbb{R}^{+} \times \mathbb{R}^{+} \times \mathbb{R}\right)$, т. е. $K$ удовлетворяет условию Каратеодори по третьему аргументу на множестве $\mathbb{R}^{+} \times \mathbb{R}^{+} \times \mathbb{R}$, последнее означает, что функция $K$ при каждом фиксированном $\tau \in \mathbb{R}$ измерима по $(x, t) \in \mathbb{R}^{+} \times \mathbb{R}^{+}$и почти при всех $(x, t) \in \mathbb{R}^{+} \times \mathbb{R}^{+}$непрерывна по $\tau$ на $\mathbb{R}$;

$3)$ для каждой измеримой функции $\varphi(x), 0 \leqslant \varphi(x) \leqslant \delta\left(\frac{1}{m_{1}} x+c\right), x>0$, функции $K(x, t, \varphi(t))$ и $\int_{0}^{\infty} K(x, t, \varphi(t)) d t$ измеримы соответственно по $t$ и $x$;

4) существует измеримая функция $\mu(x), 0 \leqslant \mu(x) \leqslant 1, x \in \mathbb{R}^{+}, \mu(x) \uparrow$ по $x$ на $\mathbb{R}^{+},(1-\mu(x)) x^{j} \in L_{1}(0,+\infty), j=0,1$, такая, что

$$
\begin{gathered}
K(x, t, \tau) \geqslant \mu(x)\left(K_{0}(x-t)-K_{0}(x+t)\right) \tau \quad \forall(x, t) \in \mathbb{R}^{+} \times \mathbb{R}^{+}, \\
0 \leqslant \tau \leqslant \delta\left(\frac{1}{m_{1}} t+c\right) ;
\end{gathered}
$$

5) $K(x, t, \tau) \uparrow$ по $\tau$ на $\left[0, \delta\left(\frac{1}{m_{1}} t+c\right)\right]$ при каждом фиксированном $(x, t) \in$ $\mathbb{R}^{+} \times \mathbb{R}^{+}$. 
Случай b). Пусть существует число $\eta>0$ такое, что:

$\left.1^{\circ}\right) \int_{0}^{\infty} K(x, t, \eta) d t \leqslant \eta, x \in \mathbb{R}^{+}$

$\left.2^{\circ}\right) \quad K \in \operatorname{Carat}\left(\mathbb{R}^{+} \times \mathbb{R}^{+} \times[0, \eta]\right)$ по $\tau$;

$3^{\circ}$ ) для каждой измеримой функции $\varphi(x), 0 \leqslant \varphi(x) \leqslant \eta, x>0$, функции $K(x, t, \varphi(t))$ и $\int_{0}^{\infty} K(x, t, \varphi(t)) d t$ измеримы соответственно по $t$ и $x$;

$\left.4^{\circ}\right) \quad K(x, t, \tau) \geqslant \mu(x)\left(K_{0}(x-t)-K_{0}(x+t)\right) \tau$ для любых точек $(x, t, \tau) \in$ $\mathbb{R}^{+} \times \mathbb{R}^{+} \times[0, \eta]$

$\left.5^{\circ}\right) \quad K(x, t, \tau) \uparrow$ по $\tau$ на $[0, \eta]$ при каждом фиксированном $(x, t) \in \mathbb{R}^{+} \times \mathbb{R}^{+}$.

Докажем следующие теоремы.

Теорема 3.1. Пусть функиия $K(x, t, \tau)$ удовлетворяет условиям 1)-5). Тогда если $\lambda=-1$ не является собственным значениям оператора $W$, то уравнение (1.1) обладает нетривиальным и неотрицательным решением $f(x)$, которое имеет следующую асимптотику: $f(x) \sim \frac{\delta}{m_{1}} x, x \rightarrow+\infty$.

Теорема 3.2. Предположим, что функиия $K(x, t, \tau)$ удовлетворяет условиям $\left.\left.1^{\circ}\right)-5^{\circ}\right)$. Тогда если $\lambda=-1$ является собственным значениям оператора $W$, то уравнение (1.1) имеет ненулевое неотрицательное ограниченное решение $f(x)$ с пределом $\lim _{x \rightarrow+\infty} f(x)=\eta$.

3.2. Доказательство теорем 3.1, 3.2. Примеры функции $K(x, t, \tau)$. Сначала докажем теоремы 3.1, 3.2.

ДокАЗАТЕЛЬСТво ТЕОРЕмы 3.1. Введем следующие последовательные приближения:

$$
\begin{gathered}
f^{(n+1)}(x)=\int_{0}^{\infty} K\left(x, t, f^{(n)}(t)\right) d t, \quad f^{(0)}(x)=\delta\left(\frac{1}{m_{1}} x+c\right), \\
n=0,1,2, \ldots, \quad x \in \mathbb{R}^{+} .
\end{gathered}
$$

По индукции докажем, что

$$
\delta\left(\frac{1}{m_{1}} x+c\right) \geqslant f^{(n)}(x) \geqslant \delta B(x), \quad x \in \mathbb{R}^{+}, \quad n=0,1,2, \ldots,
$$

где $B(x)$ - решение уравнения (2.41) в случае а) (см. теорему 2.3).

При $n=0$ соотношение (3.2) сразу следует из теоремы 2.3. Предполагая верность неравенства (3.2) для $n=p \in \mathbb{N}$, докажем его для $n=p+1$. В силу условий 1$), 4), 5)$ из (3.1) получим

$$
\begin{aligned}
f^{(p+1)}(x) & \geqslant \int_{0}^{\infty} K(x, t, \delta B(t)) d t \\
& \geqslant \mu(x) \delta \int_{0}^{\infty}\left[K_{0}(x-t)-K_{0}(x+t)\right] B(t) d t=\delta B(x), \\
f^{(p+1)}(x) & \leqslant \int_{0}^{\infty} K\left(x, t, \delta\left(\frac{1}{m_{1}} t+c\right)\right) d t \leqslant \delta\left(\frac{1}{m_{1}} x+c\right) .
\end{aligned}
$$

Следовательно, (3.2) доказано.

Докажем теперь, что $f^{(n)}(x) \downarrow$ по $n$. Неравенство $f^{(1)}(x) \leqslant f^{(0)}(x)$ следует из (3.2). Предполагая, что $f^{(n)}(x) \leqslant f^{(n-1)}(x)$, и учитывая условие 5$)$ и 
соотношение $(3.2)$, из $(3.1)$ получим $f^{(n+1)}(x) \leqslant f^{(n)}(x)$. Таким образом, последовательность функций $\left\{f^{(n)}(x)\right\}_{n=0}^{\infty}$ имеет точечный предел

$$
\lim _{x \rightarrow+\infty} f^{(n)}(x)=f(x) \geqslant \delta B(x),
$$

причем так как функция $K$ удовлетворяет условию 2), то из теоремы Б. Леви следует, что $f(x)$ удовлетворяет уравнению (1.1). Из (3.2) получаем следующую двойную оценку для решения:

$$
\delta B(x) \leqslant f(x) \leqslant \delta\left(\frac{1}{m_{1}} x+c\right), \quad x \in \mathbb{R}^{+} .
$$

Поскольку $B(x) \sim \frac{1}{m_{1}} x, x \rightarrow+\infty$, из (3.4) следует, что

$$
f(x) \sim \frac{\delta}{m_{1}} x, \quad x \rightarrow+\infty .
$$

Таким образом, теорема доказана.

ДокАЗАТЕЛЬСТво теОРЕмы 3.2. Рассмотрим последовательные приближения

$$
f^{(n+1)}(x)=\int_{0}^{\infty} K\left(x, t, f^{(n)}(t)\right) d t, \quad f^{(0)}(x) \equiv \eta, \quad n=0,1,2, \ldots, \quad x>0,
$$

и аналогично по индукции докажем следующее:

$$
f^{(n)}(x) \downarrow \text { по } n, \quad f^{(n)}(x) \geqslant \frac{B(x)}{\sup _{x \in \mathbb{R}^{+}} B(x)} \eta, \quad n=0,1,2, \ldots
$$

Отсюда следует существование предельной функции $f(x)=\lim _{n \rightarrow \infty} f^{(n)}(x)$, которая удовлетворяет уравнению (1.1) и двойному неравенству

$$
\frac{B(x)}{\sup _{x \in \mathbb{R}^{+}} B(x)} \eta \leqslant f(x) \leqslant \eta, \quad x \in \mathbb{R}^{+} .
$$

Поскольку $B(x) \uparrow$ по $x$ (см. теорему 2.4), из (3.8) следует существование предела $\lim _{x \rightarrow+\infty} f(x)=\eta$. Тем самым, теорема доказана.

Приведем несколько примеров функции $K(x, t, \tau)$ для случаев а) и $\mathrm{b})$.

ПримеР 3.1. В случае а) рассмотрим следующий класс функций:

$$
K(x, t, \tau)=\mu(x)\left[K_{0}(x-t)-K_{0}(x+t)\right] U(t, \tau),
$$

где $U(t, \tau)$ - определенная на $\mathbb{R}^{+} \times \mathbb{R}$ измеримая функция, причем предполагается, что существует число $\delta>0$ такое, что:

(i) $U(t, \tau) \geqslant \tau, 0 \leqslant \tau \leqslant \delta\left(\frac{1}{m_{1}} t+c\right), t>0$;

(ii) $U(t, \tau) \uparrow$ по $\tau$ на $\left[0, \delta\left(\frac{1}{m_{1}} t+c\right)\right]$ при каждом фиксированном $t>0$;

(iii) $U \in \operatorname{Carat}\left(\mathbb{R}^{+} \times \mathbb{R}\right)$ по аргументу $\tau$;

(iv) $U\left(t, \delta\left(\frac{1}{m_{1}} t+c\right)\right)=\delta\left(\frac{1}{m_{1}} t+c\right), t>0$. 
В качестве $U(t, \tau)$ можно рассматривать следующие функции:

$$
\begin{gathered}
U(t, \tau)=\left(\delta\left(\frac{1}{m_{1}} t+c\right)\right)^{1-\alpha} \tau^{\alpha}, \quad \alpha \in(0,1) \\
U(t, \tau)=\sqrt{\delta\left(\frac{1}{m_{1}} t+c\right) \tau \exp \left\{\frac{\tau}{\delta\left(\frac{1}{m_{1}} t+c\right)}-1\right\}} \\
U(t, \tau)=\tau+\frac{1}{2 \delta\left(\frac{1}{m_{1}} t+c\right)}\left(\tau-\delta\left(\frac{1}{m_{1}} t+c\right)\right)^{2}\left(1-e^{-\tau}\right) .
\end{gathered}
$$

ПримеР 3.2. В случае а) можно также рассмотреть следующий класс функций:

$$
K(x, t, \tau)=R(x, t, \tau)\left[K_{0}(x-t)-K_{0}(x+t)\right] U(t, \tau),
$$

где $R(x, t, \tau)$ - определенная на $\mathbb{R}^{+} \times \mathbb{R}^{+} \times \mathbb{R}$ измеримая функция, причем предполагается, что существует число $\delta>0$ такое, что:

(i) $R(x, t, \tau) \uparrow$ по $\tau$ на $\left[0, \delta\left(\frac{1}{m_{1}} t+c\right)\right]$ при каждом фиксированном $(x, t) \in$ $\mathbb{R}^{+} \times \mathbb{R}^{+}$

(ii) $\mu(x) \leqslant R(x, t, \tau) \leqslant 1,(x, t) \in \mathbb{R}^{+} \times \mathbb{R}^{+}, \tau \in\left[0, \delta\left(\frac{1}{m_{1}} t+c\right)\right]$;

(iii) $R \in \operatorname{Carat}\left(\mathbb{R}^{+} \times \mathbb{R}^{+} \times \mathbb{R}\right)$ по аргументу $\tau$.

Например, свойствами $R$ обладает функция

$$
R(x, t, \tau)=\frac{1-\mu(x)}{2} N(t, \tau)+\frac{1+\mu(x)}{2},
$$

где $N(t, \tau) \uparrow$ по $\tau$ на $\left[0, \delta\left(\frac{1}{m_{1}} t+c\right)\right]$ при каждом фиксированном $t>0$, справедливо двойное неравенство $0 \leqslant N(t, \tau) \leqslant 1, t>0, \tau \in\left[0, \delta\left(\frac{1}{m_{1}} t+c\right)\right]$ и $N \in \operatorname{Carat}\left(\mathbb{R}^{+} \times \mathbb{R}\right)$ по аргументу $\tau$.

ПримеР 3.3. В случае b) рассмотрим класс функций

$$
K(x, t, \tau)=\mu(x)\left[K_{0}(x-t)-K_{0}(x+t)\right] G(\tau),
$$

где $G(\tau)$ - определенная на $\mathbb{R}$ измеримая функция, причем существует число $\eta>0$ такое, что

$$
\begin{aligned}
& G \in C[0, \eta] ; \quad G \uparrow \text { по } \tau \text { на }[0, \eta] ; \\
& G(\tau) \geqslant \tau, \quad \tau \in[0, \eta] ; \quad G(\eta)=\eta .
\end{aligned}
$$

В качестве $G$ выберем следующие функции:

$$
\begin{gathered}
G(\tau)=\eta e^{\frac{\tau}{\eta}-1} ; \quad G(\tau)=\tau+\sin \tau, \quad \eta=\pi \\
G(\tau)=\eta e^{-\frac{(\tau-\eta)^{2}}{2}}, \quad \eta \in(0, \sqrt{2}) ; \quad G(\tau)=\eta \sqrt{\frac{\tau}{\eta}} .
\end{gathered}
$$

ПримеР 3.4. В случае b) рассмотрим также следующий класс функций:

$$
K(x, t, \tau)=L(x, \tau)\left[K_{0}(x-\tau)-K_{0}(x+\tau)\right] G(\tau),
$$

где $L(x, \tau)$ - измеримая функция на $\mathbb{R}^{+} \times \mathbb{R}$, причем существует число $\eta>0$ такое, что:

(i) $L(x, \tau) \uparrow$ по $\tau$ на $[0, \eta]$ при каждом фиксированном $x>0$;

(ii) $\mu(x) \leqslant L(x, \tau) \leqslant\left(\int_{-\infty}^{x} K_{0}(\tau) d \tau\right)^{-1},(x, \tau) \in \mathbb{R}^{+} \times[0, \eta]$;

(iii) $L \in \operatorname{Carat}\left(\mathbb{R}^{+} \times[0, \eta]\right)$. 


\section{§ 4. Решение уравнения (1.1) в случае нелинейной миноранты для оператора Урысона. Сильная нелинейность}

\section{1. Некоторые априорные оценки для решений вспомогательных} линейных уравнений. Рассмотрим следующее неоднородное интегральное уравнение:

$$
\xi(x)=2 \omega(x+A)+\mu(x) \int_{0}^{\infty}\left[K_{0}(x-t)-\varepsilon K_{0}(x+t)\right] \xi(t) d t, \quad x \in \mathbb{R}^{+},
$$

относительно искомой функции $\xi(x)$. Здесь $\omega(x)$ - измеримая функция на интервале $(-\infty,+\infty)$, причем существует число $A>0$ такое, что

$$
\begin{aligned}
\omega(x) \downarrow & \text { по } x \text { на }[A,+\infty), \\
\omega(x) \in L_{1}(0,+\infty) \cap C[0,+\infty), & m_{1}(\omega) \equiv \int_{0}^{\infty} x \omega(x) d x<+\infty, \\
\omega(x) \geqslant 0, & x \in[A,+\infty) .
\end{aligned}
$$

Предположим, что функции $\mu$ и $K_{0}$ удовлетворяют соответственно условиям $(2.42)$ и $(2.2)-(2.4)$, а $l_{0} \equiv \sup _{x \in \mathbb{R}^{+}} \frac{\int_{x}^{\infty} K_{0}(\tau) d \tau}{K_{0}(x)}<+\infty$. Из результатов работы [16] следует, что уравнение имеет неотрицательное и ограниченное решение: $2 \omega(x+A) \leqslant \xi(x) \in M(0,+\infty)$. Введем множество

$$
\Pi \equiv\left[\max \left(\frac{\max \left(\varkappa, \gamma_{0}\right)}{\beta_{0}}, A\right),+\infty\right),
$$

где $\varkappa=\sup _{x \in \mathbb{R}^{+}} \xi(x)$, а $\gamma_{0} \geqslant A-$ некоторое фиксированное число, для которого $\omega\left(\gamma_{0}\right)<\gamma_{0}$.

Существование такого числа непосредственно следует из (4.2)-(4.4).

Лемма 4.1. Пусть $\gamma \in \Pi$ - некоторое число. Тогда как в случае а), так и в случае b) имеем

$$
B_{\gamma}(x) \equiv \gamma B^{*}(x) \geqslant \xi(x), \quad x \in \mathbb{R}^{+} .
$$

ДокАЗАТЕЛЬСтво. Поскольку $\gamma \in \Pi$, из соотношения (4.5) вытекает, что

$$
\gamma \geqslant \frac{\max \left(\varkappa, \gamma_{0}\right)}{\beta_{0}}
$$

Следовательно, из теорем 2.3, 2.4 в случаях а) и b) получим

$$
B_{\gamma}(x)=\gamma B^{*}(x) \geqslant \gamma \beta_{0} \geqslant \max \left(\varkappa, \gamma_{0}\right) \geqslant \varkappa \geqslant \xi(x) .
$$

Тем самым, лемма доказана.

Введем функцию

$$
\lambda_{\gamma}(x)=1-\frac{\omega\left(x+B_{\gamma}(x)\right)}{B_{\gamma}(x)}, \quad x \in \mathbb{R}^{+} .
$$


ЛЕмма 4.2. Функция $\lambda_{\gamma}(x)$ обладает следующими свойствами:

1) $0<\lambda_{\gamma}(x) \leqslant 1, x \in \mathbb{R}^{+}$;

2) $\left(1-\lambda_{\gamma}(x)\right) x^{j} \in L_{1}(0,+\infty), j=0,1$;

3) $\lambda_{\gamma}(x) \uparrow$ по $x$ на $\mathbb{R}^{+}$;

4) $\lim _{x \rightarrow+\infty} \lambda_{\gamma}(x)=1$.

ДоказАтельство. Сначала докажем 1). Пусть $\gamma \in \Pi$; тогда $\gamma \geqslant \frac{\max \left(\varkappa, \gamma_{0}\right)}{\beta_{0}}$ и, следовательно, $B_{\gamma}(x) \geqslant \gamma_{0} \geqslant A$. Поскольку $\omega \downarrow$ на $[A,+\infty)$, то

$$
\omega\left(x+B_{\gamma}(x)\right) \leqslant \omega\left(x+\gamma_{0}\right) .
$$

Итак,

$$
\lambda_{\gamma}(x) \geqslant 1-\frac{\omega\left(x+\gamma_{0}\right)}{B_{\gamma}(x)} \geqslant 1-\frac{\omega\left(x+\gamma_{0}\right)}{\gamma_{0}} \geqslant 1-\frac{\omega\left(\gamma_{0}\right)}{\gamma_{0}}>0, \quad \lambda_{\gamma}(x) \leqslant 1 .
$$

Теперь докажем 2). Имеем

$$
x^{j}(1-\lambda(x)) \leqslant \frac{x^{j}}{A} \omega\left(x+\gamma_{0}\right) \in L_{1}(0,+\infty), \quad j=0,1 .
$$

Свойства 3), 4) непосредственно следуют из (4.6). Лемма доказана.

Пусть $r(x)$ удовлетворяет следующему неоднородному интегральному уравнению:

$r(x)=2 \omega\left(x+B_{\gamma}(x)\right)+\lambda_{\gamma}(x) \mu(x) \int_{0}^{\infty}\left[K_{0}(x-t)-\varepsilon K_{0}(x+t)\right] r(t) d t, \quad x \in(0,+\infty)$.

Введем простые последовательные приближения для уравнения (4.7):

$$
\begin{gathered}
r^{(n+1)}(x)=2 \omega\left(x+B_{\gamma}(x)\right)+\lambda_{\gamma}(x) \mu(x) \int_{0}^{\infty}\left[K_{0}(x-t)-\varepsilon K_{0}(x+t)\right] r^{(n)}(t) d t, \\
r^{(0)}(x)=2 \omega\left(x+B_{\gamma}(x)\right), \quad n=0,1,2, \ldots, \quad x>0 .
\end{gathered}
$$

Индукцией по $n$ можно доказать, что

$$
r^{(n)}(x) \uparrow \text { по } n, \quad r^{(n)}(x) \leqslant \xi(x), \quad n=0,1,2, \ldots
$$

Таким образом, последовательность функций $\left\{r^{(n)}(x)\right\}_{n=0}^{\infty}$ имеет точечный предел

$$
\lim _{n \rightarrow \infty} r^{(n)}(x)=r(x),
$$

причем по теореме Б. Леви предельная функция $r(x)$ удовлетворяет уравнению (4.7), а из (4.8) следует также, что

$$
2 \omega\left(x+B_{\gamma}(x)\right) \leqslant r(x) \leqslant \xi(x) \leqslant B_{\gamma}(x), \quad x \in(0,+\infty), \quad \gamma \in \Pi .
$$

Рассмотрим теперь однородное уравнение

$$
q(x)=\lambda_{\gamma}(x) \mu(x) \int_{0}^{\infty}\left[K_{0}(x-t)-\varepsilon K_{0}(x+t)\right] q(t) d t, \quad x>0 .
$$


Заметим, что функция

$$
\tilde{q}_{\gamma}(x)=2 B_{\gamma}(x)-r(x), \quad x>0,
$$

удовлетворяет уравнению (4.10). Действительно, имеем

$$
\begin{aligned}
\lambda_{\gamma}(x) & \mu(x) \int_{0}^{\infty}\left[K_{0}(x-t)-\varepsilon K_{0}(x+t)\right] \tilde{q}_{\gamma}(t) d t \\
& =\left(1-\frac{\omega\left(x+B_{\gamma}(x)\right)}{B_{\gamma}(x)}\right) \mu(x) \int_{0}^{\infty}\left[K_{0}(x-t)-\varepsilon K_{0}(x+t)\right]\left(2 B_{\gamma}(t)-r(t)\right) d t \\
& =2 B_{\gamma}(x)-2 \omega\left(x+B_{\gamma}\right)-\lambda(x) \mu(x) \int_{0}^{\infty}\left[K_{0}(x-t)-\varepsilon K_{0}(x+t)\right] r(t) d t \\
& =2 B_{\gamma}(x)-r(x)=\tilde{q}_{\gamma}(x) .
\end{aligned}
$$

Введем следующие последовательные функции:

$$
\begin{gathered}
q^{(n+1)}(x)=\lambda_{\gamma}(x) \mu(x) \int_{0}^{\infty}\left[K_{0}(x-t)-\varepsilon K_{0}(x+t)\right] q^{(n)}(t) d t \\
q^{(0)}(x)=2 B_{\gamma}(x), \quad n=0,1,2, \ldots, \quad x>0 .
\end{gathered}
$$

Индукцией по $n$ докажем, что

$$
q^{(n)}(x) \downarrow \quad \text { по } n .
$$

В случае $n=1$ имеем

$$
q^{(1)}(x)=2 \lambda_{\gamma}(x) B_{\gamma}(x) \leqslant 2 B_{\gamma}(x)=q^{(0)}(x),
$$

так как $\lambda_{\gamma}(x) \leqslant 1$. Предполагая, что $q^{(n)}(x) \leqslant q^{(n-1)}(x)$, из (4.12) получаем $q^{(n+1)}(x) \leqslant q^{(n)}(x)$. Заметим, что

$$
q^{(n)}(x) \leqslant 2 \lambda_{\gamma}(x) B_{\gamma}(x), \quad n=1,2,3, \ldots .
$$

Теперь докажем, что

$$
q^{(n)}(x) \geqslant \tilde{q}_{\gamma}(x), \quad n=0,1,2, \ldots
$$

В случае $n=0$ неравенство (4.14) непосредственно следует из представления (4.11) функции $\tilde{q}_{\gamma}(x)$. Предполагая, что $q^{(n)}(x) \geqslant \tilde{q}_{\gamma}(x)$ при некотором $n \in \mathbb{N}$, из (4.12) получим

$$
q^{(n+1)}(x) \geqslant \lambda_{\gamma}(x) \mu(x) \int_{0}^{\infty}\left[K_{0}(x-t)-K_{0}(x+t)\right] \tilde{q}_{\gamma}(t) d t=\tilde{q}_{\gamma}(x) .
$$

Таким образом, из (4.13), (4.14) следует существование точечного предела последовательности $\left\{q^{(n)}(x)\right\}_{n=0}^{\infty}$ :

$$
\lim _{n \rightarrow \infty} q^{(n)}(x)=q(x) \leqslant 2 \lambda_{\gamma}(x) B_{\gamma}(x),
$$

причем $q(x)$ удовлетворяет уравнению (4.10) и цепочке неравенств

$$
B_{\gamma}(x) \leqslant \tilde{q}_{\gamma}(x) \leqslant q(x) \leqslant 2 \lambda_{\gamma}(x) B_{\gamma}(x) \leqslant 2 B_{\gamma}(x) .
$$


Неравенство $\tilde{q}_{\gamma}(x) \geqslant B_{\gamma}(x)$ следует из леммы 4.1 и (4.9). Нетрудно убедиться в том, что если $q(x)$ является решением уравнения (4.10) и удовлетворяет неравенствам (4.16), то функция

$$
\psi(x)=\frac{q(x)}{\lambda_{\gamma}(x)}
$$

будет удовлетворять уравнению

$$
\psi(x)=\mu(x) \int_{0}^{\infty}\left[K_{0}(x-t)-K_{0}(x+t)\right] \lambda_{\gamma}(t) \psi(t) d t
$$

и неравенствам

$$
B_{\gamma}(x) \leqslant \tilde{q}_{\gamma}(x) \leqslant q(x) \leqslant \psi(x) \leqslant 2 B_{\gamma}(x), \quad \gamma \in \Pi .
$$

Это будет использовано в следующем пункте.

4.2. Однопараметрическое семейство положительных решений для одного класса нелинейных интегральных уравнений типа Гаммерштейна. В этом пункте докажем следующий результат, с помощью которого выведем основные теоремы 4.3, 4.4 настоящей работы.

Теорема 4.1. Предположим, что функиии $K_{0}, \mu$ и $\omega$ удовлетворяют условиям (2.2)-(2.4), (2.42) и (4.2)-(4.4) соответственно, причем $l_{0}<+\infty, \varepsilon \in$ $[0,1)$. Тогда уравнение Гаммерштейна

$$
f(x)=\mu(x) \int_{0}^{\infty}\left[K_{0}(x-t)-\varepsilon K_{0}(x+t)\right](f(t)-\omega(t+f(t))) d t, \quad x>0,
$$

обладает однопараметрическим семейством $\left\{f_{\gamma}(x)\right\}_{\gamma \in \Pi}$ положительньх решений со следующими свойствами:

1) $f_{\gamma}(x) \uparrow$ по $x$ на $\mathbb{R}^{+}, \gamma \in \Pi$,

2) если $\gamma_{1}, \gamma_{2} \in \Pi u \gamma_{1}>\gamma_{2}$, mo $f_{\gamma_{1}}(x)-f_{\gamma_{2}}(x) \geqslant 2\left(\gamma_{1}-\gamma_{2}\right) \beta_{0}$,

3) $\psi(x) \leqslant f_{\gamma}(x) \leqslant 2 B_{\gamma}(x), \gamma \in \Pi, x \in \mathbb{R}^{+}$, причем:

а) если $\lambda=-1$ не является собственным значением $W$, то $f_{\gamma}(x) \sim \frac{2 \gamma}{m_{1}} x$, при $x \rightarrow+\infty$;

b) если $\lambda=-1$ является собственным значением оператора $W$, mо $f_{\gamma}(x) \in$ $M(0,+\infty), \gamma \in \Pi$.

ДокАЗАтЕльство. Введем следующие специальные последовательные функции:

$$
\begin{gathered}
f^{(n+1)}(x)=\mu(x) \int_{0}^{\infty}\left[K_{0}(x-t)-\varepsilon K_{0}(x+t)\right]\left(f^{(n)}(t)-\omega\left(t+f^{(n)}(t)\right)\right) d t \\
f^{(0)}(x)=2 B_{\gamma}(x), \quad n=0,1,2, \ldots, \quad x>0 .
\end{gathered}
$$

Индукцией по $n$ нетрудно убедиться в том, что:

(i) $f^{(n)}(x) \downarrow$ по $n$;

(ii) $f^{(n)}(x) \geqslant \psi(x), n=0,1,2, \ldots$; 
(iii) если $\gamma_{1}, \gamma_{2} \in \Pi$ и $\gamma_{1}>\gamma_{2}$, то $f_{\gamma_{1}}^{(n)}(x)-f_{\gamma_{2}}^{(n)}(x) \geqslant 2\left(B_{\gamma_{1}}(x)-B_{\gamma_{2}}(x)\right)$, $n=0,1,2, \ldots$;

(iv) $f^{(n)}(x) \uparrow$ по $x$ на $(0,+\infty), n=0,1,2, \ldots$.

Сначала по индукции докажем (ii). Пусть $n=0$; тогда неравенство (ii) следует из (4.17). Предположим, что (ii) справедливо при некотором $n \in \mathbb{N}$; тогда с учетом монотонности $\omega$ на $[A,+\infty)$ и очевидных неравенств $\psi(x) \geqslant$ $B_{\gamma}(x) \geqslant A$ из (4.19) получим

$$
\begin{aligned}
f^{(n+1)}(x) & \geqslant \mu(x) \int_{0}^{\infty}\left[K_{0}(x-t)-\varepsilon K_{0}(x+t)\right](\psi(t)-\omega(t+\psi(t))) d t \\
& \geqslant \mu(x) \int_{0}^{\infty}\left[K_{0}(x-t)-K_{0}(x+t)\right]\left(\psi(t)-\omega\left(t+B_{\gamma}(t)\right)\right) d t \\
& =\mu(x) \int_{0}^{\infty}\left[K_{0}(x-t)-K_{0}(x+t)\right]\left(\psi(t)-\left(1-\lambda_{\gamma}(t)\right) B_{\gamma}(t)\right) d t \\
& \geqslant \mu(x) \int_{0}^{\infty}\left[K_{0}(x-t)-K_{0}(x+t)\right]\left(\psi(t)-\left(1-\lambda_{\gamma}(t)\right) \psi(t)\right) d t=\psi(x)
\end{aligned}
$$

Теперь докажем (i). Действительно, так как $f^{(n)}(x) \geqslant \psi(x) \geqslant A$, то с учетом свойств функции $\omega$ на $[A,+\infty)$ из (4.19) получим

$$
f^{(1)}(x) \leqslant 2 \mu(x) \int_{0}^{\infty}\left[K_{0}(x-t)-\varepsilon K_{0}(x+t)\right] B_{\gamma}(t) d t=2 B_{\gamma}(x)=f^{(0)}(x) .
$$

Предполагая, что $f^{(n)}(x) \leqslant f^{(n-1)}(x)$, и учитывая $f^{(n)}(x) \geqslant A, n=0,1,2, \ldots$, из (4.19) приходим к неравенству $f^{(n+1)}(x) \leqslant f^{(n)}(x)$.

Докажем теперь (iii). При $n=0$ неравенство из (iii) преобразуется в очевидное равенство. Предположим, что (iii) выполняется при некотором $n \in \mathbb{N}$. Тогда из (4.19) имеем

$$
\begin{aligned}
& f_{\gamma_{1}}^{(n+1)}(x)-f_{\gamma_{2}}^{(n+1)}(x)=\mu(x) \int_{0}^{\infty}\left[K_{0}(x-t)-K_{0}(x+t)\right]\left(f_{\gamma_{1}}^{(n)}(t)-f_{\gamma_{2}}^{(n)}(t)\right) d t \\
& \quad+\mu(x) \int_{0}^{\infty}\left[K_{0}(x-t)-K_{0}(x+t)\right]\left[\omega\left(t+f_{\gamma_{2}}^{(n)}(t)\right)-\omega\left(t+f_{\gamma_{1}}^{(n)}(t)\right)\right] d t \\
& \quad \geqslant 2\left(B_{\gamma_{1}}(x)-B_{\gamma_{2}}(x)\right),
\end{aligned}
$$

так как $\omega \downarrow$ на $[A,+\infty)$.

Докажем (iv). В случае $n=0$ это следует из монотонности $B_{\gamma}(x)$ (см. теоремы $2.3,2.4)$. Предположим, что $f^{(m)}(x) \uparrow$ по $x$ на $(0,+\infty), m \in \mathbb{N}$, и докажем, что $f^{(m+1)}(x) \uparrow$ по $x$ на $(0,+\infty)$. Пусть $x_{1}, x_{2}>0, x_{1}>x_{2},-$ произвольные числа. Тогда, записывая (4.19) в виде

$$
\begin{aligned}
f^{(m+1)}(x)=\mu(x) \int_{-\infty}^{x} K_{0}(\tau) & \left(f^{(m)}(x-\tau)-\omega\left(x-\tau+f^{(m)}(\tau)\right)\right) d \tau \\
& +\mu(x) \int_{0}^{\infty} K_{0}(x+t)\left(f^{(m)}(t)-\omega\left(t+f^{(m)}(t)\right)\right) d t
\end{aligned}
$$

и учитывая монотонность функций $\omega, f^{(m)}$ и $K_{0}$, получим

$$
f^{(m+1)}\left(x_{1}\right)-f^{(m+1)}\left(x_{2}\right) \geqslant 0 .
$$

Следовательно, (iv) установлено. 
Из (i) и (ii) получаем точечную сходимость функциональной последовательности $\left\{f^{(n)}(x)\right\}_{n=0}^{\infty}: \lim _{n \rightarrow \infty} f^{(n)}(x)=f_{\gamma}(x) \geqslant \psi(x)$, причем с помощью теоремы Б. Леви можно доказать, что $f_{\gamma}(x)$ удовлетворяет уравнению (4.18), а из (iv), (iii) получим

$$
\begin{gathered}
f_{\gamma}(x) \uparrow \quad \text { по } x \text { на }(0,+\infty), \\
f_{\gamma_{1}}(x)-f_{\gamma_{2}}(x) \geqslant 2\left(B_{\gamma_{1}}(x)-B_{\gamma_{2}}(x)\right) \geqslant 2\left(\gamma_{1}-\gamma_{2}\right) \beta_{0} .
\end{gathered}
$$

В случае а) из свойства 3) с учетом (4.17) приходим к асимптотическому равенству $f_{\gamma}(x) \sim \frac{2 \gamma}{m_{1}} x$, когда $x \rightarrow+\infty$. В случае b) из неравенства $f_{\gamma}(x) \leqslant 2 B_{\gamma}(x) \in$ $M(0,+\infty)$ следует, что $f_{\gamma} \in M(0,+\infty)$. Теорема доказана.

Отметим, что уравнение (4.18) в случае $\varepsilon=0, \mu \equiv 1$ ранее исследовалось в [18].

Теорема 4.2. Пусть $\stackrel{\omega}{(t, \tau)}$ - определенная на $\mathbb{R}^{+} \times \mathbb{R}$ вещественная функиия, причем существует число $A>0$ такое, что:

1) $\dot{\omega} \in \operatorname{Carat}\left(\mathbb{R}^{+} \times[A,+\infty)\right)$ по аргументу $\tau$;

2) $\stackrel{\omega}{\omega}(t, \tau) \downarrow$ по $\tau$ на $[A,+\infty)$ при каждом фиксированном $x \in \mathbb{R}^{+}$;

3) $\stackrel{\omega}{\omega}(t, \tau) \leqslant \omega(t+\tau),(t, \tau) \in \mathbb{R}^{+} \times[A,+\infty)$.

Тогда уравнение Гаммерштейна

$$
F(x)=\mu(x) \int_{0}^{\infty}\left[K_{0}(x-t)-K_{0}(x+t)\right](F(t)-\grave{\omega}(t, F(t))) d t, \quad x>0,
$$

обладает однопараметрическим семейством $\left\{F_{\gamma}(x)\right\}_{\gamma \in \Pi}$ положительных решений со следующими свойствами:

(i) если $\stackrel{\iota}{\downarrow}$ nо $t$ на $\mathbb{R}^{+}$, то функция $F_{\gamma}(x)$ монотонно возрастает по $x$ на $\mathbb{R}^{+}, \gamma \in \Pi_{0} \equiv\left[\max \left(\frac{\max \left(\varkappa, \gamma_{0}\right)}{\beta_{0}}, A\right),+\infty\right)$,

(ii) если $\gamma_{1}, \gamma_{2} \in \Pi_{0}, \gamma_{1}>\gamma_{2}$, mo $F_{\gamma_{1}}(x)-F_{\gamma_{2}}(x) \geqslant 2\left(\gamma_{1}-\gamma_{2}\right) \beta_{0}$,

(iii) $f_{\gamma}(x) \leqslant F_{\gamma}(x) \leqslant 2 B_{\gamma}(x), \gamma \in \Pi$, причем:

а) если $\lambda=-1$ не является собственным значением оператора $W$, то $F_{\gamma}(x) \sim \frac{2 \gamma}{m_{1}} x, x \rightarrow+\infty$

b) если $\lambda=-1$ является собственным значением оператора $W$, то $F_{\gamma} \in$ $M(0,+\infty)$.

ДокАЗАТЕЛьСТво аналогично доказательству теоремы 4.1.

4.3. Решение основного уравнения (1.1). Справедливы следующие теоремы.

Теорема 4.3. Пусть существует число $\delta \geqslant 2 \max \left(\frac{\max \left(\varkappa, \gamma_{0}\right)}{\beta_{0}}, A\right)$ maкое, чmo:

1) при каждом фиксированном $(x, t) \in \mathbb{R}^{+} \times \mathbb{R}^{+}$имеем $K(x, t, \tau) \uparrow$ по $\tau$ на множестве $\left[A, \delta\left(\frac{1}{m_{1}} t+c\right)\right]$, где числа $m_{1}$ и с задаются согласно $(2.22),(2.26)$;

2) $K \in \operatorname{Carat}\left(\mathbb{R}^{+} \times \mathbb{R}^{+} \times[A,+\infty)\right)$ по аргументу $\tau$ и для каждой измеримой функиии $\varphi(x), 0 \leqslant \varphi(x) \leqslant \delta\left(\frac{1}{m_{1}} x+c\right), x>0$, функции $K(x, t, \varphi(t))$ u $\int_{0}^{\infty} K(x, t, \varphi(t)) d t$ измеримь соответственно по $t u x$; 
3) $K(x, t, \tau) \geqslant \mu(x)\left[K_{0}(x-t)-\varepsilon K_{0}(x+t)\right](\tau-\omega(t+\tau))$ для любъх $(x, t) \in$ $\mathbb{R}^{+} \times \mathbb{R}^{+}, \tau \in\left[A, \delta\left(\frac{1}{m_{1}} t+c\right)\right]$, где функиии $K_{0}, \mu$ и ям (2.2)-(2.4), (2.42) и (4.2)-(4.4) соответственно, причем $l_{0}<+\infty, \varepsilon \in[0,1)$;

4) при $x \in \mathbb{R}^{+}$

$$
\frac{1}{\frac{1}{m_{1}} x+c} \int_{0}^{\infty} K\left(x, t, \delta\left(\frac{1}{m_{1}} t+c\right)\right) d t \leqslant \delta .
$$

Тогда если $\lambda=-1$ не является собственным значением оператора $W$, то уравнение (1.1) имеет положительное решение $\varphi(x)$ с асимптотикой $\varphi(x) \sim$ $\frac{\delta}{m_{1}} x$, когда $x \rightarrow+\infty$.

Теорема 4.4. Предположим, что число $\lambda=-1$ является собственным значением оператора $W$ и существует число

$$
\eta \geqslant 2 \sup _{x \in \mathbb{R}^{+}} B^{*}(x) \max \left(\frac{\max \left(\varkappa, \gamma_{0}\right)}{\beta_{0}}, A\right)
$$

maкое, что:

1) при каждом фиксированном $(x, t) \in \mathbb{R}^{+} \times \mathbb{R}^{+}$имеем $K(x, t, \tau) \uparrow$ по $\tau$ на отрезке $[A, \eta]$;

2) $K \in \operatorname{Carat}\left(\mathbb{R}^{+} \times \mathbb{R}^{+} \times[A, \eta]\right)$ по аргументу $\tau$ и для каждой измеримой функции $\varphi(x), 0 \leqslant \varphi(x) \leqslant \eta, x>0$, функции $K(x, t, \varphi(t)) u \int_{0}^{\infty} K(x, t, \varphi(t)) d t$ измеримы соответственно по $t u x$;

3) $K(x, t, \tau) \geqslant \mu(x)\left[K_{0}(x-t)-\varepsilon K_{0}(x+t)\right](\tau-\omega(t+\tau))$ для всех $(x, t, \tau) \in$ $\mathbb{R}^{+} \times \mathbb{R}^{+} \times[A, \eta]$, где функиии $K_{0}, \mu$ и (2.42) и (4.2)-(4.4) соответственно, причем $\varepsilon \in[0,1)$;

4) $\int_{0}^{\infty} K(x, t, \eta) d t \leqslant \eta$ для любого $x \in \mathbb{R}^{+}$.

Тогда уравнение (1.1) обладает положительным и ограниченным решением $\varphi(x)$, причем $\varphi(x) \leqslant \eta$.

ДокАЗАТЕЛЬСтво теоремы 4.3. Согласно теореме 4.1 уравнение (4.18) об-

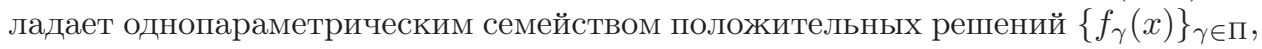
причем в случае, когда $\lambda=-1$ не является собственным значением оператора $W$, эти решения имеют асимптотику $f_{\gamma}(x) \sim \frac{2 \gamma}{m_{1}} x, x \rightarrow+\infty$, и удовлетворяют двойному неравенству $A \leqslant f_{\gamma}(x) \leqslant 2 \gamma\left(\frac{1}{m_{1}} x+c\right), x \in \mathbb{R}^{+}$. Выберем из множества П число $\gamma^{*}=\frac{\delta}{2}$. Этому числу соответствует решение $f_{\gamma^{*}}(x)$, обладающее свойствами

$$
A \leqslant f_{\gamma^{*}}(x) \leqslant \delta\left(\frac{1}{m_{1}} x+c\right) ; \quad f_{\gamma^{*}}(x) \sim \frac{\delta}{m_{1}} x, \quad x \rightarrow+\infty .
$$

Рассмотрим следующие последовательные функции:

$$
\begin{gathered}
\varphi^{(n+1)}(x)=\int_{0}^{\infty} K\left(x, t, \varphi^{(n)}(t)\right) d t, \quad \varphi_{0}(x)=\delta\left(\frac{1}{m_{1}} x+c\right), \\
n=0,1,2, \ldots, \quad x>0 .
\end{gathered}
$$

Используя свойства 1)-4) из условия теоремы для функции $K$, докажем, что

$$
A \leqslant f_{\gamma^{*}}(x) \leqslant \varphi^{(n)}(x) \leqslant \delta\left(\frac{1}{m_{1}} x+c\right), \quad n=0,1,2, \ldots
$$


В случае $n=0$ соотношение (4.23) непосредственно следует из (4.21). Предположим, что (4.23) имеет место при некотором $n \in \mathbb{N}$, и докажем его при $n+1$. Используя свойства функции $K$, из (4.22) получим

$$
\begin{aligned}
& \varphi^{(n+1)}(x) \leqslant \int_{0}^{\infty} K\left(x, t, \delta\left(\frac{1}{m_{1}} t+c\right)\right) d t \leqslant \delta\left(\frac{1}{m_{1}} x+c\right), \\
\varphi^{(n+1)}(x) \geqslant & \int_{0}^{\infty} K\left(x, t, f_{\gamma^{*}}(t)\right) d t \\
\geqslant & \mu(x) \int_{0}^{\infty}\left[K_{0}(x-t)-\varepsilon K_{0}(x+t)\right]\left(f_{\gamma^{*}}(t)-\omega\left(t+f_{\gamma^{*}}(t)\right)\right) d t=f_{\gamma^{*}}(x) .
\end{aligned}
$$

Теперь докажем, что

$$
\varphi^{(n)}(x) \downarrow \quad \text { по } n .
$$

Неравенство $\varphi^{(1)}(x) \leqslant \varphi^{(0)}(x)$ сразу следует из соотношения (4.23). Предполагая, что $\varphi^{(n)}(x) \leqslant \varphi^{(n-1)}(x)$, и учитывая монотонность $K$ по $\tau$ из $(4.22)$, получим неравенство $\varphi^{(n+1)}(x) \leqslant \varphi^{(n)}(x)$.

Таким образом, из (4.23), (4.24) следует, что последовательность функций $\left\{\varphi^{(n)}(x)\right\}_{n=0}^{\infty}$ имеет точечный предел

$$
\begin{gathered}
\lim _{n \rightarrow \infty} \varphi^{(n)}(x)=\varphi(x), \\
f_{\gamma^{*}}(x) \leqslant \varphi(x) \leqslant \delta\left(\frac{1}{m_{1}}+c\right),
\end{gathered}
$$

причем из предельной теоремы Б. Леви следует, что $\varphi(x)$ удовлетворяет уравнению (1.1), а из (4.26) с учетом (4.21) получим $\varphi(x) \sim \frac{\delta}{m_{1}} x, x \rightarrow+\infty$. Теорема доказана.

ДоКАЗАТЕЛЬСтво теОРемы 4.4. Рассмотрим однопараметрическое семейство решений $\left\{f_{\gamma}(x)\right\}_{\gamma \in \Pi}$ уравнения (4.18) в случае, когда $\lambda=-1$ является собственным значением $W$. Выберем число $\tilde{\gamma}=\frac{\eta}{2 \sup _{x \in \mathbb{R}^{+}} B^{*}(x)} \in \Pi$ и решение $f_{\tilde{\gamma}}(x)$. Тогда из теоремы 4.1 следует, что

$$
A \leqslant f_{\tilde{\gamma}}(x) \leqslant 2 B_{\tilde{\gamma}}(x) \leqslant 2 \tilde{\gamma} \sup _{x \in \mathbb{R}^{+}} B^{*}(x)=\eta
$$

Рассмотрим следующие специальные последовательные функции:

$$
\varphi^{(n+1)}(x)=\int_{0}^{\infty} K\left(x, t, \varphi^{(n)}(t)\right) d t, \quad \varphi^{(0)}(x)=\eta, \quad n=0,1,2, \ldots, \quad x>0 .
$$

Аналогичным образом, как и в доказательстве теоремы 4.3, здесь также можно убедиться в том, что

$$
\varphi^{(n)}(x) \downarrow \text { по } n ; \quad \varphi^{(n)}(x) \geqslant f_{\tilde{\gamma}}(x), \quad n=0,1,2, \ldots
$$

Следовательно, существует предел $\lim _{n \rightarrow \infty} \varphi^{(n)}(x)=\varphi(x) \leqslant \eta$, который удовлетворяет уравнению (1.1). Теорема доказана. 


\section{§ 5. Примеры функций $K, K_{0}, \mu$ и $\omega$}

5.1. Примеры нелинейных интегральных уравнений. Приведем несколько примеров интегральных уравнений типа Гаммерштейна и Урысона, для которых выполняются все условия теорем 4.3, 4.4 .

Пример 5.1. В случае, когда $\lambda=-1$ не является собственным значением оператора $W$ (случай а)), рассмотрим класс уравнений

$$
\varphi(x)=\mu(x) \int_{0}^{\infty}\left[K_{0}(x-t)-\varepsilon K_{0}(x+t)\right](U(t, \varphi(t))-\stackrel{\circ}{\omega}(t, \varphi(t))) d t,
$$

где $\stackrel{\leftrightarrow}{\omega}$ удовлетворяет условиям 1$)-3)$ из теоремы 4.2 , а $U(t, \tau)$ удовлетворяет условиям (i)-(iv) из примера 3.1 для $\delta \geqslant 2 \max \left(\frac{\max \left(\varkappa, \gamma_{0}\right)}{\beta_{0}}, A\right)$.

ПримеР 5.2. В случае а) рассмотрим также следующий класс нелинейных уравнений:

$$
\varphi(x)=\int_{0}^{\infty} R(x, t, \varphi(t))\left[K_{0}(x-t)-\varepsilon K_{0}(x+t)\right](U(t, \varphi(t))-\stackrel{\circ}{\omega}(t, \varphi(t))) d t,
$$

где функция $R(x, t, \tau)$ удовлетворяет условиям (i)-(iii) из примера 3.2 для $\delta \geqslant$ $2 \max \left(\frac{\max \left(\varkappa, \gamma_{0}\right)}{\beta_{0}}, A\right)$.

ПримеР 5.3. В случае, когда $\lambda=-1$ является собственным значением оператора $W$ (случай $\mathrm{b}))$, рассмотрим класс уравнений

$$
\varphi(x)=\mu(x) \int_{0}^{\infty}\left[K_{0}(x-t)-\varepsilon K_{0}(x+t)\right](G(\varphi(t))-\stackrel{\leftrightarrow}{\omega}(t, \varphi(t))) d t,
$$

где $G$ удовлетворяет условиям (3.10), (3.11) для

$$
\eta \geqslant \eta_{0} \equiv 2 \sup _{x \in \mathbb{R}^{+}} B^{*}(x) \max \left(\frac{\max \left(\varkappa, \gamma_{0}\right)}{\beta_{0}}, A\right) .
$$

ПримеР 5.4. В случае b) рассмотрим также следующий класс нелинейных уравнений:

$$
\varphi(x)=\int_{0}^{\infty} L(x, \varphi(t))\left[K_{0}(x-t)-\varepsilon K_{0}(x+t)\right](G(\varphi(t))-\stackrel{\circ}{\omega}(t, \varphi(t))) d t,
$$

где $L(x, \tau)$ удовлетворяет условиям (i)-(iii) из примера 3.4 для $\eta \geqslant \eta_{0}$. В качестве функции $\stackrel{\omega}{ }(t, z)$ можно рассмотреть следующий класс функций:

$$
\stackrel{\circ}{\omega}(t, z)=h(z) \omega(t+z),
$$

где $h$ - измеримая функция на $\mathbb{R}$, причем $h \in C[A,+\infty), h \downarrow$ по $z$ на $[A,+\infty)$, $0 \leqslant h(z) \leqslant 1, z \in[A,+\infty)$. 
5.2. Примеры ядра $K_{0}$ и функции $\mu$. Приведем несколько примеров функций $K_{0}$, для которых выполняются условия (2.2)-(2.4), а также условие $l_{0}<+\infty$.

ПримеР 5.5. Рассмотрим функцию

$$
K_{0}(x)=\frac{1}{\sqrt{\pi}} e^{-x^{2}}, \quad x \in \mathbb{R} .
$$

Для функции (5.5), очевидно, выполняются условия (2.2)-(2.4). Докажем, что $l_{0} \equiv \sup _{x \in \mathbb{R}^{+}} \frac{\int_{x}^{\infty} K_{0}(\tau) d \tau}{K_{0}(x)}<+\infty$. Рассмотрим функцию

$$
\psi(x)=e^{x^{2}} \int_{x}^{\infty} e^{-t^{2}} d t, \quad x \in \mathbb{R}^{+} .
$$

Заметим, что

$$
\psi(0)=\frac{\sqrt{\pi}}{2}, \quad \psi^{\prime}(x)=2 x e^{x^{2}} \int_{x}^{\infty} e^{-t^{2}} d t-1 \leqslant e^{x^{2}}\left(\int_{x}^{\infty} e^{-t^{2}} d t^{2}-e^{-x^{2}}\right)=0 .
$$

Следовательно, $\psi \downarrow$ на $\mathbb{R}^{+}$. С другой стороны, используя правило Лопиталя, получим

$$
\psi(+\infty)=0
$$

Таким образом, из изложенного следует, что в случае ядра (5.5) получаем $l_{0}=\frac{\sqrt{\pi}}{2}$. Используя формулу $m_{1}=\sqrt{\frac{1}{2} \nu_{2}}$ (см. [19]), где $\nu_{2}=\int_{0}^{\infty} x^{2} K_{0}(x) d x$, в случае ядра (5.5) получаем $m_{1}=\frac{1}{2}$.

ПримеР 5.6. Теперь рассмотрим следующую функцию:

$$
K_{0}(x)=\int_{a}^{b} e^{-|x| s} d \sigma(s), \quad 0<a<b \leqslant+\infty,
$$

где $\sigma \uparrow$ на $[a, b)$, причем $2 \int_{a}^{b} \frac{1}{s} d \sigma(s)=1$. Тогда

$$
l_{0}=\sup _{x \in \mathbb{R}^{+}} \frac{\int_{a}^{b} \frac{1}{s} e^{-x s} d \sigma(s)}{\int_{a}^{b} e^{-x s} d \sigma(s)} \leqslant \frac{1}{a}<+\infty
$$

a $m_{1}=\sqrt{\frac{1}{2} \nu_{2}}$, где $\nu_{2}=\int_{0}^{\infty} K_{0}(x) x^{2} d x=2 \int_{a}^{b} \frac{1}{s^{3}} d \sigma(s)$.

ПримеР 5.7. В качестве $\mu(x)$ можно рассмотреть следующую функцию:

$$
\mu(x)=1-\theta_{0} e^{-\theta_{1} x}, \quad x \in \mathbb{R}^{+},
$$

где $\theta_{0}, \theta_{1}>0, \theta_{0}<1$. Тогда $\mu_{0}=1-\theta_{0}>0$.

На одном частном примере функции $K_{0}$ выясним, является ли число $\lambda=-1$ собственным значением оператора $W$. Пусть $K_{0}(x)=\frac{1}{2} e^{-|x|}$. Тогда (см. [16]) ядра имеют вид $v_{ \pm}(x)=e^{-x}, W(x)=2 \varepsilon e^{-x}$. Докажем, что $\lambda=-1$ не является собственным значением оператора $W$ с ядром $W(x)=2 \varepsilon e^{-x}, \varepsilon \in[0,1]$. Предположим обратное: существует нетривиальное решение уравнения

$$
\rho(x)=-2 \varepsilon \int_{0}^{\infty} e^{-(x+t)} \rho(t) d t
$$

в одном из банаховых пространств $E$. 
Из (5.6) непосредственно следует, что

$$
\rho_{0}(1+\varepsilon)=0, \quad \rho_{0}=\int_{0}^{\infty} e^{-t} \rho(t) d t .
$$

Поскольку $\varepsilon \in[0,1]$, то $\rho_{0}=0$ и, следовательно, $\rho \equiv 0$ в силу (5.6). Таким образом, $\lambda=-1$ не является собственным значением вполне непрерывного оператора $W$, т. е. для ядра $K_{0}(x)=\frac{1}{2} e^{-|x|}$ мы можем использовать теоремы 3.1 , $4.3,4.1, \mathrm{~b}), 4.2, \mathrm{~b})$.

\section{§6. Однопараметрическое семейство положительных решений для соответствующего неоднородного интегрального уравнения Гаммерштейна. Сильная нелинейность для неоднородных уравнений}

6.1. Построение однопараметрического семейства положительных решений. В настоящем параграфе мы построим однопараметрическое семейство положительных решений для следующего класса нелинейных неоднородных интегральных уравнений типа Гаммерштейна:

$$
\begin{gathered}
h(x)=g(x)+\int_{0}^{\infty}\left[K_{0}(x-t)-\varepsilon K_{0}(x+t)\right] R_{0}(x, h(t))(h(t)-\stackrel{\circ}{\omega}(t, h(t))) d t, \\
x \in \mathbb{R}^{+} \equiv[0,+\infty),
\end{gathered}
$$

относительно искомой вещественной функции $h(x)$. Здесь $K_{0}$ и $\dot{\omega}$ удовлетворяют соответственно условиям (2.2)-(2.4) и (4.2)-(4.4), а $R_{0}(x, \tau)$ - определенная на $\mathbb{R}^{+} \times \mathbb{R}$ вещественная функция, причем:

(A) существует число $A>0$ такое, что $R_{0}(x, \tau) \uparrow$ по $\tau$ на $[A,+\infty)$ при каждом фиксированном $x \in \mathbb{R}^{+}$;

(B) $R_{0} \in \operatorname{Carat}\left(\mathbb{R}^{+} \times[A,+\infty)\right)$ по аргументу $\tau$;

(C) $\mu(x) \leqslant R_{0}(x, \tau) \leqslant 1,(x, \tau) \in \mathbb{R}^{+} \times[A,+\infty)$, где $\mu(x)$ удовлетворяет условиям (2.42).

Свободный член $g(x)$, определенный на $\mathbb{R}^{+}$, является положительной функцией, удовлетворяющей неравенству

$$
c_{0} \equiv \sup _{x \in \mathbb{R}^{+}} \frac{g(x)}{\int_{x}^{\infty} K(\tau) d \tau}<+\infty .
$$

\section{Справедлива}

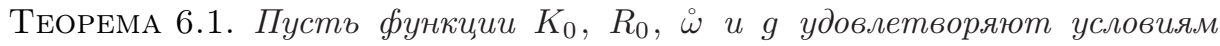
(2.2)-(2.4), (A)-(C), 1)-3) из теоремы 4.2 и (6.2) соответственно. Тогда:

а) если $\lambda=-1$ не является собственным значением $W$ u $l_{0}<+\infty$, то в этом случае уравнение (6.1) обладает однопараметрическим семейством положительных решений $\left\{h_{\gamma}(x)\right\}_{\gamma \in \Pi_{1}}$, причем:

(i) при всяком $\gamma \in \Pi_{1}$ имеют место асимптотика $h_{\gamma}(x) \sim \frac{2 \gamma}{m_{1}} x, x \rightarrow+\infty$, и неравенство $h_{\gamma}(x) \geqslant \varphi_{\gamma}(x), x \in \mathbb{R}^{+}$;

(ii) если $\gamma_{1}, \gamma_{2} \in \Pi_{1}, \gamma_{1}>\gamma_{2}, u R_{0} \equiv 1$, mo $h_{\gamma_{1}}(x)-h_{\gamma_{2}}(x) \geqslant 2\left(\gamma_{1}-\gamma_{2}\right) \beta_{0}$;

(iii) $h_{\gamma}(x) \leqslant 2 \gamma\left(\frac{1}{m_{1}} x+c_{0}+\max \left(\frac{l_{0}}{m_{1}}, \frac{2 r_{0}}{m_{1}}, \sup _{x \in \mathbb{R}^{+}}|Q(x)|\right)\right), \gamma \in \Pi_{1}$, zде $\Pi_{1}=$ $\left[\max \left(\frac{\max \left(\varkappa, \gamma_{0}\right)}{\beta_{0}}, A, \frac{1}{2}\right),+\infty\right)$; 
b) если $\lambda=-1$ является собственным значением оператора $W$, то уравнение (6.1) обладает однопараметрическим семейством положительных решений $\left\{h_{\gamma}(x)\right\}_{\gamma \in \Pi_{0}}$, әде $\Pi_{0}=\left[\max \left(\frac{\max \left(\varkappa, \gamma_{0}\right)}{\beta_{0}}, A, \frac{c_{0}}{2 c}\right),+\infty\right)$, причем:

(i) при всяком $\gamma \in \Pi_{0} \subset \Pi$ имеют место включение $h_{\gamma} \in M(0,+\infty)$ и неравенство $h_{\gamma}(x) \geqslant \varphi_{\gamma}(x), x \in \mathbb{R}^{+}$;

(ii) если $\gamma_{1}, \gamma_{2} \in \Pi_{0}, \gamma_{1}>\gamma_{2}, u R_{0} \equiv 1$, mo $h_{\gamma_{1}}(x)-h_{\gamma_{2}}(x) \geqslant 2\left(\gamma_{1}-\gamma_{2}\right) \beta_{0}$;

(iii) $\lim _{x \rightarrow+\infty} h_{\gamma}(x)=2 \gamma c$.

ДоказАтельство. Докажем, например, утверждение а). Доказательство утверждения b) более простое.

Рассмотрим следующие последовательные функции:

$$
\begin{gathered}
h^{(n+1)}(x)=g(x)+\int_{0}^{\infty}\left[K_{0}(x-t)-\varepsilon K_{0}(x+t)\right] R_{0}\left(x, h^{(n)}(t)\right) \\
\times\left(h^{(n)}(t)-\omega\left(t, h^{(n)}(t)\right)\right) d t, \\
h^{(0)}(x)=2 \gamma\left(\frac{1}{m_{1}} x+c_{0}+\max \left(\frac{l_{0}}{m_{1}}, \frac{2 r_{0}}{m_{1}}, \sup _{x \in \mathbb{R}^{+}}|Q(x)|\right)\right), \\
n=0,1,2, \ldots, \quad x \in \mathbb{R}^{+} .
\end{gathered}
$$

Сначала индукцией по $n$ докажем, что

$$
\begin{gathered}
\varphi_{\gamma}(x) \leqslant h^{(n)}(x) \leqslant 2 \gamma\left(\frac{1}{m_{1}} x+c_{0}+\max \left(\frac{l_{0}}{m_{1}}, \frac{2 r_{0}}{m_{1}}, \sup _{x \in \mathbb{R}^{+}}|Q(x)|\right)\right), \\
n=0,1,2, \ldots, \quad x \in \mathbb{R}^{+} .
\end{gathered}
$$

При $n=0$ соотношение (6.4) следует из (6.3). Предполагая верность двойного неравенства (6.4) при $n=p \in \mathbb{N}$, докажем его в случае $n=p+1$. Обозначая

$$
\tilde{c}=c_{0}+\max \left(\frac{l_{0}}{m_{1}}, \frac{2 r_{0}}{m_{1}}, \sup _{x \in \mathbb{R}^{+}}|Q(x)|\right),
$$

получим

$$
\begin{aligned}
h^{(p+1)}(x) \leqslant & c_{0} \int_{x}^{\infty} K_{0}(t) d t+2 \gamma \int_{0}^{\infty}\left[K_{0}(x-t)-\varepsilon K_{0}(x+t)\right]\left(\frac{1}{m_{1}} x+\tilde{c}\right) d t \\
= & c_{0} \int_{x}^{\infty} K_{0}(t) d t+2 \gamma\left(\int_{-\infty}^{x} K_{0}(\tau)\left(\frac{1}{m_{1}}(x-\tau)+\tilde{c}\right) d \tau\right. \\
& \left.\quad-\varepsilon \int_{x}^{\infty} K_{0}(\tau)\left(\frac{1}{m_{1}}(x-\tau)+\tilde{c}\right) d \tau\right) \\
= & c_{0} \int_{x}^{\infty} K_{0}(\tau) d \tau+2 \gamma\left(\int_{-\infty}^{+\infty} K_{0}(\tau)\left(\frac{1}{m_{1}}(x-\tau)+\tilde{c}\right) d \tau\right. \\
& \left.\quad-\int_{x}^{\infty} K_{0}(\tau)\left(\frac{1}{m_{1}}(x-\tau)+\tilde{c}\right) d \tau-\varepsilon \int_{x}^{\infty} K_{0}(\tau)\left(\frac{1}{m_{1}}(\tau-x)+\tilde{c}\right) d \tau\right) \\
= & c_{0} \int_{x}^{\infty} K_{0}(\tau) d \tau+2 \gamma\left(\frac{1}{m_{1}} x+\tilde{c}-\left(\frac{1-\varepsilon}{m_{1}} x+\tilde{c}\right) \int_{x}^{\infty} K_{0}(\tau) d \tau\right) \\
& \quad+\frac{2 \gamma \varepsilon}{m_{1}} \int_{x}^{\infty} K_{0}(\tau) \tau d \tau \\
\leqslant & 2 \gamma\left(\frac{1}{m_{1}} x+\tilde{c}\right),
\end{aligned}
$$


так как справедлива оценка (2.34). С другой стороны, имеем

$$
\begin{aligned}
h^{(p+1)}(x) & \geqslant g(x)+\mu(x) \int_{0}^{\infty}\left[K_{0}(x-t)-\varepsilon K_{0}(x+t)\right]\left(\varphi_{\gamma}(t)-\omega\left(t, \varphi_{\gamma}(t)\right)\right) d t \\
& \geqslant \mu(x) \int_{0}^{\infty}\left[K_{0}(x-t)-\varepsilon K_{0}(x+t)\right]\left(\varphi_{\gamma}(t)-\omega\left(t, \varphi_{\gamma}(t)\right)\right) d t=\varphi_{\gamma}(x) .
\end{aligned}
$$

Аналогичным образом, как и при доказательстве теоремы 4.1, устанавливается, что

$$
\begin{gathered}
h^{(n)}(x) \downarrow \quad \text { по } n, \\
\text { если } R_{0} \equiv 1, \text { то } h_{\gamma_{1}}^{(n)}-h_{\gamma_{2}}^{(n)} \geqslant 2\left(\gamma_{1}-\gamma_{2}\right) B(x), \quad n=0,1,2, \ldots .
\end{gathered}
$$

Следовательно, существует предел

$$
\lim _{n \rightarrow \infty} h^{(n)}=h(x),
$$

который по теореме Б. Леви удовлетворяет уравнению (6.1). Используя теорему 4.1 и двойное неравенство

$$
\varphi_{\gamma}(x) \leqslant h_{\gamma}(x) \leqslant \frac{2 \gamma}{m_{1}} x+\tilde{c}
$$

приходим к асимптотике $h_{\gamma} \sim \frac{2 \gamma x}{m_{1}}, x \rightarrow+\infty, \gamma \in \Pi_{1}$. Из (6.6) следует также неравенство $h_{\gamma_{1}}-h_{\gamma_{2}} \geqslant 2\left(\gamma_{1}-\gamma_{2}\right) \beta_{0}$. Теорема доказана.

С использованием теоремы 6.1 аналогичным образом, как в п. 4.3, устанавливаются следующие результаты.

Теорема 6.2. Пусть существует иисло $\eta \geqslant \eta_{0} \equiv 2 c \max \left(\frac{\max \left(\varkappa, \gamma_{0}\right)}{\beta_{0}}, A, \frac{c_{0}}{2 c}\right)$ maкoе, чmo:

1) $K(x, t, \tau) \uparrow$ по $\tau$ на отрезке $[A, \eta]$ при каждом биксированном $(x, t) \in$ $\mathbb{R}^{+} \times \mathbb{R}^{+}$

2) $K \in \operatorname{Carat}\left(\mathbb{R}^{+} \times \mathbb{R}^{+} \times[A, \eta]\right)$ по аргументу $\tau$ и для каждой измеримой

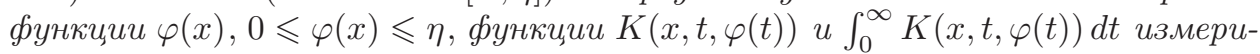
мы соответственно по $t$ и $x$;

3) $K(x, t, \tau) \geqslant \mu(x)\left[K_{0}(x-t)-\varepsilon K_{0}(x+t)\right](\tau-\omega(t+\tau)),(x, t, \tau) \in \mathbb{R}^{+} \times \mathbb{R}^{+} \times$ $[A, \eta]$, где $\mu, K_{0}$ и $\omega_{0}$ удовлетворяют соответственно условиям (2.2)-(2.4), (A)-(C) $u(4.2)-(4.4)$;

4) $\int_{0}^{\infty} K(x, t, \eta) d t \leqslant \eta, x \in \mathbb{R}^{+}$.

Тогда если $\lambda=-1$ является собственным значением оператора $W, a$ $g(x) \geqslant 0$ удовлетворяет условию (6.2), то уравнение Урысона

$$
H(x)=g(x)+\int_{0}^{\infty} K(x, t, H(t)) d t, \quad x>0,
$$

имеет положительное и ограниченное решение, причем $H(x) \leqslant \eta$. 
Теорема 6.3. Пусть существует число $\delta \geqslant \delta_{0} \equiv 2 \max \left(\frac{\max \left(\varkappa, \gamma_{0}\right)}{\beta_{0}}, A, \frac{1}{2}\right)$ maкое, что:

1) $K(x, t, \tau) \uparrow$ nо $\tau$ для $A \leqslant \tau \leqslant \delta\left(\frac{1}{m_{1}} t+\tilde{c}\right)$ при кажсдом фиксированном $(x, t) \in \mathbb{R}^{+} \times \mathbb{R}^{+}$

2) $K \in \operatorname{Carat}\left(\mathbb{R}^{+} \times \mathbb{R}^{+} \times[A,+\infty)\right)$ по аргументу $\tau$ и для каждой измеримой функции $\varphi(x), 0 \leqslant \varphi(x) \leqslant \delta\left(\frac{1}{m_{1}} x+c\right)$, функции $K(x, t, \varphi(t)) u \int_{0}^{\infty} K(x, t, \varphi(t)) d t$ измеримы соответственно по $t$ и $x$;

3) $K(x, t, \tau) \geqslant \mu(x)\left[K_{0}(x-t)-\varepsilon K_{0}(x+t)\right](\tau-\omega(t+\tau)),(x, t) \in \mathbb{R}^{+} \times \mathbb{R}^{+}$, $A \leqslant \tau \leqslant \delta\left(\frac{1}{m_{1}} t+\tilde{c}\right)$, где $\mu, K_{0}$ u $\omega_{0}$ удовлетворяют соответственно условиям (2.2)-(2.4), (A)-(C) и (4.2)-(4.4), причем $l_{0}<+\infty$;

4) при $x \in \mathbb{R}^{+}$

$$
\frac{1}{\frac{1}{m_{1}} x+\tilde{c}} \int_{0}^{\infty} K\left(x, t, \delta\left(\frac{1}{m_{1}} t+\tilde{c}\right)\right) d t \leqslant \delta .
$$

Тогда если $\lambda=-1$ не является собственным значением оператора $W u$ функиия $g(x) \geqslant 0$ удовлетворяет условию (6.2), то уравнение (6.7) имеет положительное решение с асимптотикой $H(x) \sim \frac{\delta}{m_{1}} x, x \rightarrow+\infty$.

Отметим, что приведенные примеры 3.1-3.4 функции $K(x, t, \tau)$ в случае $\eta \geqslant \eta_{0}$ (или $\left.\delta \geqslant \delta_{0}\right)$ удовлетворяют также всем условиям теоремы 6.2 (или теоремы 6.3).

В заключение автор выражает глубокую благодарность Н. Б. Енгибаряну за обсуждения результатов, а также рецензенту за полезные замечания.

\section{Список литературы}

1. М.А. Красносельский, Положителъные решения операторных уравнений, Физматлит, М., 1962.

2. П. П. Забрейко, "О непрерывности и полной непрерывности операторов П. С. Урысона”, Докл. АН СССР, 161 (1965), 1007-1010; англ.пер.: P.P. Zabrejko, "Continuity and complete continuity of Urysohn operators", Soviet Math. Dokl., 6 (1965), 540-544.

3. М. А. Красносельский, "Признаки непрерывности некоторых нелинейных операторов", Укр. матем. журн., 2:3 (1950), 70-86.

4. G. Emmanuele, "An existence theorem for Hammerstein integral equations", Portugal. Math., 51:4 (1994), 607-611.

5. H. Brezis, F. E. Browder, "Existence theorems for nonlinear integral equations of Hammerstein type", Bull. Amer. Math. Soc., 81:1 (1975), 73-78.

6. П. П. Забрейко, "О непрерывности нелинейного оператора", Сиб. матем. журн., 5:4 (1964), 958-960.

7. J. Banás, "Integrable solutions of Hammerstein and Urysohn integral equations", $J$. Austral. Math. Soc. Ser. A, 46:1 (1989), 61-68.

8. Х.А. Хачатрян, “Достаточные условия разрешимости интегрального уравнения Урысона на полуоси", Докл. РАН, 425:4 (2009), 462-465; англ. пер.: Kh. A. Khachatryan, "Sufficient conditions for the solvability of the Urysohn integral equation on a half-line", Dokl. Math., 79:2 (2009), 246-249.

9. А. Х. Хачатрян, Х. А. Хачатрян, "Об одном нелинейном интегральном уравнении типа уравнения Гаммерштейна с некомпактным оператором", Матем. сб., 201:4 (2010), 125-136; англ. пер.: A. Kh. Khachatryan, Kh. A. Khachatryan, "A nonlinear 
integral equation of Hammerstein type with a noncompact operator", Sb. Math., 201:4 (2010), 595-606.

10. Kh. A. Khachatryan, "On solvability of some classes of Urysohn nonlinear integral equations with noncompact operators", Уфимск. матем. журн., 2:2 (2010), 102-117.

11. Х. А. Хачатрян, "Разрешимость одного класса интегро-дифференциальных уравнений второго порядка с монотонной нелинейностью на полуоси", Изв. РАН. Сер. матем., 74:5 (2010), 191-204; англ. пер.: Kh. A. Khachatryan, "Solubility of a class of second-order integro-differential equations with monotone non-linearity on a semi-axis", Izv. Math., 74:5 (2010), 1069-1082.

12. И. Ц. Гохберг, И. А. Фельдман, Уравнения в свертках и проекиионные методы их решения, Наука, М., 1971; англ. пер.: I. C. Gohberg, I. A. Fel'dman, Convolution equations and projection methods for their solution, Amer. Math. Soc., Providence, RI, 1974.

13. С. Г. Крейн, Линейные уравнения в банаховом пространстве, Наука, М., 1971.

14. Н.Б. Енгибарян, Л.Г. Арабаджян, "О некоторых задачах факторизации для интегральных операторов типа свертки”, Дифферени. уравнения, 26:1 (1990), 1442-1452; англ. пер.: N. B. Engibaryan, L. G. Arabadzhyan, "Some factorization problems for convolution integral operators", Differential Equations, 26:8 (1990), 1069-1078.

15. А.Н. Колмогоров, В.С. Фомин, Элементы теории функиий и функционального анализа, 5-е изд., Наука, М., 1981; англ. пер. 2-го изд.: А. N. Kolmogorov, S. V. Fomin, Introductory real analysis, Prentice-Hall, Englewood Cliffs, NJ, 1970.

16. Г. Г. Геворкян, Н.Б. Енгибарян, "Новые теоремы для интегрального уравнения восстановления", Изв. НАН Армении. Математика, 32:1 (1997), 5-20; англ. пер.: G. G. Gevorkyan, N. B. Engibaryan, "New theorems for the renewal integral equation", J. Contemp. Math. Anal., 32:1 (1997), 2-16.

17. Л. Г. Арабаджян, "Об одном интегральном уравнении теории переноса в неоднородной среде", Дифферени. уравнения, 23:9 (1987), 1618-1622.

18. Л.Г. Арабаджян, "Решения одного интегрального уравнения типа Гаммерштейна”, Изв. НАН Армении. Математика, 32:1 (1997), 21-28; англ. пер.: L. G. Arabadzhyan, "Solutions of certain integral equations of the Hammerstein type", J. Contemp. Math. Anal., 32:1 (1997), 17-24.

19. Л. Г. Арабаджян, Н. Б. Енгибарян, "Уравнения в свертках и нелинейные функциональные уравнения", Итоги науки и техн. Сер. Матем. анализ, 22, ВИНИТИ, M., 1984, 175-244; англ. пер.: L. G. Arabadzhyan, N. B. Engibaryan, "Convolution equations and nonlinear functional equations", J. Soviet Math., 36:6 (1987), 745-791.

X. А. Хачатрян (Кн. А. Кнachatryan)

Институт математики НАН Армении, г. Ереван

E-mail: Khach82@rambler.ru
Поступило в редакцию 29.09.2010 09.03.2011 